\title{
Goodwill Can Hurt: a Theoretical and Experimental Investigation of Return Policies in Auctions
}

\author{
By C. Bram Cadsby, Ninghua Du, Ruqu Wang and Jun Zhang*
}

Will generous return policies in auctions benefit bidders? We investigate this issue using second-price common-value auctions. Theoretically, we find that the bidding equilibrium is unique unless returns are free, in which case there exist multiple equilibria with different implications for sellers. Moreover, more generous return policies hurt bidders by eroding consumer surplus through higher bids. In the experiment, bids increase and bidders' earnings decrease with more generous return policies as predicted. With free returns, many bidders bid above the highest possible value, subsequently returning the item regardless of value. Though consistent with equilibrium behavior, this is not optimal for sellers.

\section{Department of Economics and Finance University of Guelph Discussion Paper 2015-01}

\footnotetext{
*Cadsby: Department of Economics and Finance, University of Guelph, 50 Stone Road East, Guelph, ON N1G 2W1, Canada (e-mail: bcadsby@uoguelph.ca)

Du: Key Laboratory of Mathematical Economics and School of Economics, Shanghai University of Finance and Economics, Shanghai 200433, P.R. China (e-mail: ninghua.du@mail.shufe.edu.cn)

Wang: Department of Economics, Queen's University, Kingston, ON K7L 3N6, Canada (e-mail: wangr@queensu.ca)

Zhang: Economics Discipline Group, School of Business, University of Technology Sydney, Sydney, NSW, Australia (e-mail: Jun.Zhang-1@uts.edu.au)

Acknowledgments. The authors benefited from constructive comments by Jacob Goeree, Joseph Taoyi Wang, Philippos Louis, Maros Servatka, workshop participants at the ESEI Center for Market Design, University of Zurich, and the School of Economics and Management, Tsinghua University. Du thanks Zhibo Xu and Guanfu Fang for excellent research assistance, and the Key Laboratory of Mathematical Economics at Shanghai University of Finance and Economics and the Chinese Ministry of Education for financial support.
} 


\section{Introduction}

The rapid growth of Internet commerce has resulted in the development of online auctions as a popular trading method over the past decades. Return policies are widely available in such online auctions. Return policies permit auction winners to change their minds by paying a pre-specified penalty fee when they receive relevant ex-post information after the auction concludes. A recent search for antique auctions on eBay.com yielded 35,758 such auctions with 23,014 (64\%) of the sellers offering a 7-day or 14-day money-back guarantee. The percentage of art auctions offering refunds on eBay.com was even higher, with 131,944 out of 175,329 sellers offering a money-back guarantee, representing $75 \%$ of art auctions.

Return policies are sometimes observed in traditional auctions as well. For example, deposits required in auctions for valuable objects such as spectrum licenses, oil field leases, and mineral and gas rights can be treated as fixed-fee return policies. If an auction winner fails to pay his/her full bid upon winning, then the deposit is not refunded. For example, shortly after the conclusion of the 1996 "C-block" radio frequency spectrum auction in the U.S., the bidders re-evaluated the market values of the licenses they had just won and determined that the values were far less than the 10-billion-dollar winning bids that they were required to pay. As a result, several bidders declined to make their payments to the Federal Communications Commission, and thus forfeited their deposits. 
How would a return policy affect bidders' behavior and what kind of return policy would most benefit them? What kind of return policy would most benefit sellers? How should a revenue-maximizing seller select the optimal return policy? These are some of the issues we will investigate in this paper. We focus on the common-value model in Wilson [20], which fits reasonably well with auctions for oil field leases, gas and mineral rights, and spectrum licenses. Our model should also be informative for auctions of objects with a major common-value component, such as art and antiques. ${ }^{1}$

We analyze the behavior of bidders in second-price auctions and focus on linear return policies where the seller can charge a percentage fee in addition to a fixed fee. Linear return policies are very popular because they are, like linear pricing, easy to implement in practice. We provide a closed-form solution for the unique equilibrium when returns are not completely free. When returns are free, there exist multiple equilibria, all of which yield zero payoffs for the bidders, but have different implications for the seller.

Results from the literature on return policies offered by retail stores, such as Che [3], predict that consumers will be better off with a more generous return policy. However, perhaps surprisingly, it turns out that a more generous return policy actually hurts consumers in auctions. This counterintuitive result arises from the fact that a more generous return policy not only protects consumers from bad shocks, but also induces

${ }^{1}$ Resale can introduce a common-value component to a private-value good. (See Haile [9], for an example.) 
them to bid more aggressively in the auction, resulting in higher bids and lower consumer surplus.

We also examine how return policies affect the seller's revenue. On the one hand, with a more generous return policy, bidders bid more aggressively, which enhances the seller's revenue. On the other hand, a more generous return policy makes it more likely that the winner will return the object. By selecting an appropriate return policy, the seller can achieve higher revenue by balancing the trade-off between higher bids and fewer returns.

We find that the optimal (linear) return policy should always be in the form of a fixed fee (or subsidy), implying that the seller should not charge a percentage fee. This resembles many return policies in reality: deposits in oil field leases, mineral and gas rights, and spectrum auctions are usually specified in fixed amounts, and many sellers on eBay provide money-back guarantees with fixed shipping subsidies or shipping and handling fees.

We conduct an experiment to test the predictions of our theory. In the experimental setting, items may have a high value of 100 or a low value of 0 , with an a priori $50 \%$ probability of each outcome. We focus on return polices with fixed fees since our theory predicts that proportional fees are suboptimal for seller revenue maximization. There are four experimental treatments: No Return (NR), High Fee (HF), Low Fee (LF) and Free Return (FR). We observe that bids increase and bidders' earnings decrease when return 
policies are more generous as predicted by theory. Correspondingly, sellers' revenues increase with more generous return policies as long as some positive fee is charged for a return. However, when returns are free, many bidders bid above the highest possible value for the good, and subsequently return the item regardless of the revealed value. While this is consistent with theoretical equilibrium behavior, it is not an equilibrium that is optimal for the seller who receives zero revenue when such an outcome occurs.

This paper is related to the literature on theory of public ex-post information. When ex-post information is public and can be contracted on, its effect has long been recognized in the auction literature pioneered by Hansen [10]. In general, it has been shown that ignoring such information is sub-optimal, and adopting a mechanism conditional on the realization of the information is revenue improving. Riley [19] demonstrates that royalty bidding is better than cash bidding. Abhishek et al. [1] show that by charging an initial amount plus requiring a profit-sharing contract, the seller can generate more revenue. Demarzo et al. [5] examine bidding with securities and show that it can enhance revenue. However, all these mechanisms require the seller to track down the realized value implied by the ex-post information, which could be quite costly. In addition, sometimes the ex-post information may be unobservable, and this is common for objects sold through online auctions. In such cases, mechanisms conditional on ex-post information may not be feasible. In contrast, return policies do not require the seller to observe any ex-post information; it is solely up to the winning bidder to decide 
whether or not to return the object.

There is a huge literature on auctions. However, few papers consider return policies. Zhang [21] considers independent private values that are subject to shocks after the transaction, and illustrates how return policies can be part of an optimal mechanism. Hafalir and Yektas [8] consider second-price auctions and compare the revenues among spot auctions, forward auctions, and forward auctions with a full return policy. The information structure in their model is a special case of Zhang [21]. Our current paper considers common-value auctions with a full range of linear return policies. Huang et al. [11] recently considered an algorithm for multi-unit auctions with a partial refund for bid withdrawals that occur for exogenous reasons. That paper provides an analysis from the perspective of artificial intelligence, and thus the strategic behavior of bidders is not its focus.

In the related experimental literature, bidding in common-value auctions is well documented in laboratory settings (see Kagel and Levin [14] for a survey). Assuming symmetric bidding behavior in common-value auctions, bidders only win when they have the highest signal. Unless this is accounted for when formulating bids, the winner of the auction will receive below normal or even negative profits. Such a judgmental failure is known as the "winner's curse." Previous experimental studies show that inexperienced bidders are vulnerable to the winner's curse (Kagel and Levin [13]), while experienced bidders have learned to avoid the winner's curse by the time they appear for subsequent 
sessions (examples include Casari et al. [2], Garvin and Kagel [6] and Goertz [7]). In our study, a return policy acts as insurance against overbids and thus mitigates the winner's curse. Nonetheless, we follow the earlier experiments by introducing the factor of experience to minimize any possible impact of the winner's curse on our results.

The rest of this paper is organized as follows. In Section 2, we set up the model. In Section 3, we characterize the bidders' equilibrium strategies in second-price auctions and perform some preliminary analysis. In Section 4, we illustrate the effect of return policies on consumer surplus, social welfare and seller's revenue. In Section 5, we describe the experimental design using a simplified version of the general model. In Section 6, we discuss the experimental results. In Section 7, we conclude. All proofs are relegated to appendices.

\section{The Model}

Suppose that there are $n$ bidders bidding for one object. The object value is the same for all bidders. Let $V$ denote this common value. Assume that $V=v_{H}$ with probability $\mu_{H}$, and $V=v_{L}$ with probability $\mu_{L}=1-\mu_{H}$, where $v_{H}>v_{L}$. The distribution for $V$ is common knowledge. Before bidding starts, bidder $i \in\{1, \cdots, n\}$ receives a private signal $x_{i}$, which is correlated with $V$. However, conditional on $V$, this signal is independently distributed across the bidders. If $V=v_{H}$, then $x_{i}$ follows the distribution with c.d.f. $F_{H}(\cdot)$ and p.d.f. $f_{H}(\cdot)$. If $V=v_{L}$, then $x_{i}$ follows the distribution with c.d.f. 
$F_{L}(\cdot)$ and p.d.f. $f_{L}(\cdot)$. Assume that $F_{H}(\cdot)$ and $F_{L}(\cdot)$ have a common support $[\underline{x}, \bar{x}]$.

The object in the auction can be returned by the winning bidder for a refund. We assume that there is a shipping cost for the winning bidder to return the object. This cost is denoted by $c_{B}$. It could include the time and effort taken by the winning bidder to ship back the object as well as the actual shipping charges. Let $p$ be the transaction price (i.e., the price the winning bidder paid) in the auction. A return policy is denoted by $(\alpha, \gamma)$; if the winning bidder returns the object, s/he gets back the price s/he paid (i.e., $p$ ), minus the return fees $\alpha+\gamma p .^{2}$ Here, $\gamma$ is a prespecified proportion of the price $p$, which may correspond to a proportional restocking fee in the real world. Meanwhile, $\alpha$ is simply a fixed fee or subsidy as explained below.

We place some restrictions on the return policy to simplify the analysis. We assume that $0 \leq \gamma \leq 1$ and that $\alpha \geq-c_{B}$. The former ensures that a bidder cannot make money by simply using the win-and-return strategy. The latter ensures that the winning bidder's shipping cost is not overcompensated. If $\alpha$ is positive, then it is a fixed fee, corresponding to a handling charge or a service charge in reality; if $\alpha$ is negative, it is a subsidy, corresponding to a refund or partial refund of the winning bidder's shipping cost. The difference between the shipping $\operatorname{cost} c_{B}$ and the fixed fee $\alpha$ is that the former is paid to a third party (to cover the actual cost of shipping) while the latter is a pure transfer

\footnotetext{
${ }^{2}$ Here, we restrict our analysis to linear return fees. This simplifies the calculations significantly. Moreover, we are not aware of any other type of return policy in reality.
} 
from the winning bidder to the seller.

The game proceeds in three stages:

1. Nature selects $V=v_{H}$ or $V=v_{L}$. Conditional on $V$, each bidder receives an independent signal $(x)$.

2. A second-price auction with return policy $(\alpha, \gamma)$ is held. The winner pays accordingly and receives the object.

3. The winner learns the true $V$ and decides whether or not to return the object to the seller for a refund.

We assume that the winning bidder learns costlessly the true value of $V$ after $\mathrm{s} / \mathrm{he}$ wins and obtains the object. (The analysis is similar if $\mathrm{s} /$ he learns more but imperfect information about $V$.) In auctions for oil field leases and gas and mineral rights, for example, the winning bidders usually learn more information by doing more geological testing and analysis after winning the auction. Another example is online auctions where, once the winning bidder receives the object, s/he usually learns more about its value.

In our analysis, the likelihood ratio for bidders' signals plays an important role. Let

$$
\rho_{k}(x) \equiv \frac{k f_{H}(x) F_{H}(x)^{k-1}}{k f_{L}(x) F_{L}(x)^{k-1}}
$$

denote the likelihood ratio of $V=v_{H}$ versus $V=v_{L}$ for the highest signal among $k$ bidders. Then $\rho_{1}(x)=\frac{f_{H}(x)}{f_{L}(x)}$. Assume that $\rho_{1}(x)$ is increasing in $x$, i.e., $F_{H}$ dominates $F_{L}$ in likelihood ratio. This ensures that a higher signal implies a higher probability of $V=v_{H}$ 


\section{Equilibrium Analysis}

In this section, we characterize the bidders' equilibrium bidding function. We will focus on the symmetric perfect Bayesian equilibrium with a strictly increasing bidding function $B(\cdot)$ in the auction. We restrict our attention to bidding functions taking values in $\left[v_{L}, v_{H}\right]$. We do so because a bidder should not bid more than $v_{H}$ or less than $v_{L}$. Bidding more than $v_{H}$ sometimes gives the bidder a negative surplus and is dominated by bidding $v_{H}$. Moreover, if the bidder with the lowest possible signal bids less than $v_{L}$ in a proposed equilibrium, then it is a profitable deviation for that bidder to bid $v_{L}$ instead given that all other bidders follow the proposed equilibrium strategy. Doing so means s/he wins with positive probability and thus receives a positive expected surplus, while in the proposed equilibrium, the expected surplus was zero. Therefore bidding less than $v_{L}$ cannot be part of an equilibrium.

We will choose bidder 1 as the representative bidder in our analysis. Let $y$ denote the second highest signal among all bidders, and $z$ denote the highest signal among bidders 2, .., $n$. If $V=v_{H}$, then $y$ and $z$ follow distributions with c.d.f. $G_{H}(y)=$ $n F_{H}(y)^{n-1}-(n-1) F_{H}(y)^{n}$ and $J_{H}(z)=F_{H}(\cdot)^{n-1}$, respectively. Let $g_{H}(y)=n(n-$ 1) $f_{H}(y) F_{H}(y)^{n-2}\left[1-F_{H}(y)\right]$ and $j_{H}(z)=(n-1) f_{H}(y) F_{H}(y)^{n-2}$ denote their respective p.d.f.s. Furthermore, $G_{L}(y), J_{L}(z), g_{L}(y)$ and $j_{L}(z)$ represent similar c.d.f.s and p.d.f.s when $V=v_{L}$. 
We begin with the return stage. Suppose that bidder 1 receives signal $x$, bids $B(\tilde{x})$, and wins the object. Since it is a second-price auction, s/he pays $B(z)$. After winning, if $\mathrm{s} /$ he learns that $V=v_{H}, \mathrm{~s} /$ he will not return the object since his/her payment is less than $v_{H}$. If $\mathrm{s} /$ he learns that $V=v_{L}$, s/he returns the object for a refund if and only if $v_{L}-B(z)<-[\alpha+\gamma B(z)]-c_{B} \cdot{ }^{3}$ The left-hand side of the inequality is the payoff $\mathrm{s} / \mathrm{he}$ receives if $\mathrm{s} / \mathrm{he}$ keeps the object, while the right-hand side is the payoff $\mathrm{s} / \mathrm{he}$ receives if $\mathrm{s} /$ he returns the object for a refund.

Given the winning bidder's return decision in the return stage, we can examine the symmetric equilibrium bidding function in the auction stage. Let us first consider two hypothetical auctions. The first is a second-price auction in which no return is allowed, while the other is a second-price auction in which the winner is required to return the object when $V=v_{L}$. Let $B^{1}(x)$ and $B^{2}(x)$ denote the equilibrium bidding functions in the two hypothetical auctions. The first hypothetical auction is a standard second-price auction and a special case of Milgrom and Weber [18]. A bidder with signal $x$ bids $E\left(V \mid x_{1}=x, z=x\right)$, the expected object value conditional on his/her own signal being $x$ and the highest signal among other bidders being $z=x$. Define this value as $\Gamma(x)$. Thus,

$$
B^{1}(x)=\Gamma(x)=E\left(V \mid x_{1}=x, z=x\right)=\frac{\mu_{H} v_{H} \rho_{1}(x) \rho_{n-1}(x)+\mu_{L} v_{L}}{\mu_{H} \rho_{1}(x) \rho_{n-1}(x)+\mu_{L}} .
$$

For the second hypothetical auction, we know bidders will bid up to the amount where s/he earns zero surplus conditional on winning and paying that price (i.e., 
conditional on the highest signal from the other bidders also being $\mathrm{x}$ ). In this case, if $V=v_{H}$, s/he receives $v_{H}$; if $V=v_{L}$, s/he receives nothing but pays the proportion $\gamma$ of the price plus $\alpha+c_{B}$. As a result, the bid is the solution to

$$
\begin{aligned}
& \left(v_{H}-B\right) \mu_{H} f_{H}(x)(n-1) f_{H}(x) F_{H}(x)^{n-2} \\
& +\left(-\gamma B-\alpha-c_{B}\right) \mu_{L} f_{L}(x)(n-1) f_{L}(x) F_{L}(x)^{n-2}=0 .
\end{aligned}
$$

Therefore,

$$
B^{2}(x) \equiv \frac{\mu_{H} v_{H} \rho_{1}(x) \rho_{n-1}(x)-\left(\alpha+c_{B}\right) \mu_{L}}{\mu_{H} \rho_{1}(x) \rho_{n-1}(x)+\gamma \mu_{L}} .
$$

Note that $B^{1}(x)$ and $B^{2}(x)$ are both strictly increasing. It is useful to discuss their relationship to each other. If $\frac{\alpha+v_{L}+c_{B}}{1-\gamma}<\underline{x}, B^{1}(x)$ is always below $B^{2}(x)$; if $\frac{\alpha+v_{L}+c_{B}}{1-\gamma}>$ $\bar{x}, B^{1}(x)$ is always above $B^{2}(x)$; otherwise, $B^{1}(x)$ single crosses $B^{2}(x)$ from above at $\Gamma^{-1}\left(\frac{\alpha+v_{L}+c_{B}}{1-\gamma}\right)$. Denote $x^{*}$ as follows:

$$
x^{*}= \begin{cases}\underline{x}, & \text { if } \frac{\alpha+v_{L}+c_{B}}{1-\gamma}<\underline{x} \\ \Gamma^{-1}\left(\frac{\alpha+v_{L}+c_{B}}{1-\gamma}\right), & \text { if } \underline{x} \leq \frac{\alpha+v_{L}+c_{B}}{1-\gamma} \leq \bar{x} \\ \bar{x}, & \text { if } \frac{\alpha+v_{L}+c_{B}}{1-\gamma}>\bar{x}\end{cases}
$$

Now, we are ready to characterize the equilibrium of our model. Let $B(\cdot)$ denote the bidding function in this case. Suppose that bidder 1's signal is $x$ and $\mathrm{s} /$ he pretends to have signal $\tilde{x}$ and bids $B(\tilde{x})$. Given that $\mathrm{s} /$ he acts optimal in the return stage, his/her expected surplus in the auction is given by:

$$
\begin{aligned}
\Pi(x, \tilde{x})= & \operatorname{Pr}\left(V=v_{H} \mid x_{1}=x\right) E\left\{[V-B(z)] I\{z<\tilde{x}\} \mid x_{1}=x, V=v_{H}\right\} \\
& +\operatorname{Pr}\left(V=v_{L} \mid x_{1}=x\right) E\left\{\left[\max \left\{V-B(z),-\gamma B-\alpha-c_{B}\right\}\right]\right.
\end{aligned}
$$




$$
\begin{gathered}
\qquad\left\{\{z \tilde{x}\} \mid x_{1}=x, V=v_{L}\right\} \\
=\mu_{H}(x) \int_{\underline{x}}^{\tilde{x}}\left[v_{H}-B(y)\right] d J_{H}(z) \\
+\mu_{L}(x) \int_{\underline{x}}^{\tilde{x}}\left[\max \left\{v_{L}-B(z),-\gamma B(z)-\alpha-c_{B}\right\}\right] d J_{L}(z),
\end{gathered}
$$

where

$$
\begin{aligned}
& \mu_{H}(x) \equiv \operatorname{Pr}\left(V=v_{H} \mid x_{1}=x\right) \\
= & \frac{\operatorname{Pr}\left(x_{1}=x \mid V=v_{H}\right) \operatorname{Pr}\left(V=v_{H}\right)}{\operatorname{Pr}\left(x_{1}=x \mid V=v_{H}\right) \operatorname{Pr}\left(V=v_{H}\right)+\operatorname{Pr}\left(x_{1}=x \mid V=v_{L}\right) \operatorname{Pr}\left(V=v_{L}\right)} \\
= & \frac{f_{H}(x) \mu_{H}}{f_{H}(x) \mu_{H}+f_{L}(x) \mu_{L}}
\end{aligned}
$$

and where $\mu_{L}(x)=\operatorname{Pr}\left(V=v_{L} \mid x_{1}=x\right)=1-\mu_{H}(x)$.

In equilibrium, it is optimal for bidder 1 to report truthfully and the first order condition yields:

$$
\mu_{H}(x)\left[v_{H}-B(x)\right] j_{H}(x)+\mu_{L}(x)\left[\max \left\{v_{L}-B(x),-\gamma B(x)-\alpha-c_{B}\right\}\right] j_{L}(x)=0 .
$$

The FOC can be simplied to

$$
B(x)=\max \left\{B^{1}(x), B^{2}(x)\right\}
$$

It can also be verified that the FOC is also sufficient for the equilibrium. The proof is standard but tedious and is available in an online appendix.

We thus have the following proposition.

Proposition 1: In a second-price common-value auction with return policy $(\alpha, \gamma)$, there exists a symmetric monotone perfect Bayesian equilibrium characterized as follows. In the auction stage, bidders bid according to the strictly increasing function $B(x)=$ $\max \left\{B^{1}(x), B^{2}(x)\right\}$; and in the return stage, the winner returns the object if and only if 
$V=v_{L}$ and the second highest signal is higher than $x^{*}$. This equilibrium is unique unless $\gamma=0$ and $\alpha=-c_{B}$.

When $\gamma=0$ and $\alpha=-c_{B}$, in the equilibrium characterized above, we have $B(x)=v_{H}, \forall x$. However, this equilibrium is not unique. For example, all bidders bidding more than $v_{H}$ regardless of their signals with the winner returning the object regardless of the revealed value of $V$ is also an equilibrium. Obviously, bidding more than $v_{H}$ is weakly dominated by bidding $v_{H}$. For simplicity and continuity, when $\gamma=0$ and $\alpha=-c_{B}$, we focus in the theory on the equilibrium where $B(x)=v_{H}$ with the winning bidder always returning the object when $V=v_{L}$ and keeping it when $V=v_{H}{ }^{4}$

When the seller puts in place a no-return policy, bidders anticipate the winner's curse and adjust their bids downward from their estimates of the object's value using their own signals. When a return policy is in place, they bid more aggressively as they are somewhat protected from overbidding. In this sense, return policies mitigate the winner's curse. In fact, return policies can overdo this mitigating effect. When the return policy is generous enough, bidders may bid more than their estimates of the object's value. For example, when $\alpha=-c_{B}$ and $\gamma=0$, players will bid $v_{H}$, the highest possible value of the object. This leads to the possibility of enhancing the seller's revenue by providing a return policy. Of course, return policies can negatively impact the seller's revenue as well

\footnotetext{
${ }^{4}$ Note that our experimental result shows that if $\gamma=0$ and $\alpha=-c_{B}$, bidders actually do not follow this equilibrium prediction, but instead often play one of the weakly dominated equilibria. However, as long as there is even a very small amount of cost to return an item, the data are qualitatively consistent with our equilibrium prediction.
} 
as the efficiency of trading as the seller usually has a lower reservation value than the bidders. By selecting an appropriate return policy, the seller can achieve more revenue by balancing the tradeoff between higher bids and efficiency losses. In the following section, we will investigate this tradeoff in detail.

\section{The effects of return policies on consumer surplus, social welfare and seller's}

\section{revenue}

In this section, we first study how return policies affect bidders' expected surplus (i.e., consumer surplus) and the expected gains from trade (i.e., social welfare). We then examine the effects of return policies on the seller's revenue (i.e., producer surplus) and characterize the optimal return policy for the seller.

\subsection{Consumer surplus and social welfare}

Denote the consumer surplus as $C S(\alpha, \gamma)$, and the total surplus as $W(\alpha, \gamma)$, respectively. Let $v_{0}$ be the seller's value of the object.

Consumer surplus is given by:

$$
\begin{aligned}
& \operatorname{CS}(\alpha, \gamma) \\
& =\mu_{H}\left\{\int_{\underline{x}}^{x^{*}}\left[v_{H}-B^{1}(y)\right] g_{H}(y) d y+\int_{x^{*}}^{\bar{x}}\left[v_{H}-B^{2}(y)\right] g_{H}(y) d y\right\} \\
& \quad+\mu_{L}\left\{\int_{\underline{x}}^{x^{*}}\left[v_{L}-B^{1}(y)\right] g_{L}(y) d y+\int_{x^{*}}^{\bar{x}}\left[-a-\gamma B^{2}(y)-c_{B}\right] g_{L}(y) d y\right\} .
\end{aligned}
$$

Note that $x^{*}$ is a function of $\alpha$ and $\gamma$. The following proposition illustrates how return policies affect consumers' surplus. 
Proposition 2: With a more generous return policy (a lower $\alpha$ or $\gamma$ ), the consumer surplus is lower.

This result is somewhat counter-intuitive. In the case of return policies in retail stores, a more generous return policy protects consumers better when bad shocks occur, making them better off. This effect is also present in an auction. However, in an auction bidders are competing with each other. A more generous return policy thus induces bidders to bid more aggressively and this effect lowers consumer surplus. In our model, the second effect always dominates the first one. This is because bidders always have a higher estimate of the probability of returns in their equilibrium strategy calculation than what actually occurs. In his/her equilibrium calculation, because it is a second-price auction, a bidder assumes (correctly) that the other bidder has the same signal as him/herself when calculating his/her break-even bid. However, this bid is paid to the seller only when the other bidder has a higher signal and wins. This higher signal reduces the probability that $V=v_{L}$ and thus correspondingly reduces the probability that the winner will actually return the object relative to the probability correctly used in the equilibrium strategy calculation.

If we examine the above result from the perspective of the linkage principle, it would appear less surprising and relatively intuitive: since the return policy links bidders' payments to additional information (the true value of the object), it erases bidders' informational rents. However, the intuition is less transparent than it appears. The 
traditional linkage principal following Milgrom and Weber [18] applies only when the final allocations of the object are the same across the comparison. However, different return policies will, in general, induce different final allocations of the object. Our result suggests that the linkage principal sometimes applies even when the final allocation changes.

We can also consider how return policies would affect social welfare:

$$
W(\alpha, \gamma)=\mu_{H}\left(v_{H}-v_{0}\right)+\mu_{L}\left[\int_{x^{*}}^{\bar{x}}\left(-c_{B}\right) d G_{L}(y)+\int_{\bar{x}}^{x^{*}}\left(v_{L}-v_{0}\right) d G_{L}(y)\right]
$$

Proposition 3: With a more generous return policy (a lower $\alpha$ or $\gamma$ ), social welfare is higher if and only if $v_{L}+c_{B} \leq v_{0}$.

A more generous return policy induces more returns: this is more efficient if the seller values the returned object highly enough.

\subsection{Seller's revenue}

We will now examine the effect of the return policy on the seller's revenue and characterize the optimal linear return policy for the seller. Denote the seller's revenue as $R(\alpha, \gamma)$. It is obvious that $R(\alpha, \gamma)=W(\alpha, \gamma)-C S(\alpha, \gamma)$. If $v_{L}+c_{B} \leq v_{0}$, a more generous return policy (a lower $\alpha$ or $\gamma$ ) increases social welfare and decreases consumer surplus, therefore unambiguously increasing the seller's revenue. Note that we restrict the return policy to $\alpha \geq-c_{B}$ and $0 \leq \gamma \leq 1$. Thus, the unique optimal return policy is $\alpha=-c_{B}$ and $\gamma=0$. This is summarized in the following proposition. 
Proposition 4: If $v_{L}+c_{B} \leq v_{0}$, a more generous return policy (a lower $\alpha$ or $\gamma$ ), means that the seller's revenue is higher, implying that the optimal return policy is $\alpha=-c_{B}$ and $\gamma=0$.

The condition $v_{L}+c_{B} \leq v_{0}$ requires that the seller values the object more than bidders do when the common bidder value is low. This could be true if $v_{L}$ represents a situation where some fixable problem occurs, and it is easier for the seller than for the bidder to fix the problem. However, in general such condition could be violated.

For the rest of the analysis in this section, we will focus on the case where $v_{L}+c_{B}>$ $v_{0}$. Since $R(\alpha, \gamma)=W(\alpha, \gamma)-C S(\alpha, \gamma)$, the seller's revenue may not change monotonically with the return policy. There is no clear conclusion about how $\alpha$ and $\gamma$ would affect the seller's revenue. ${ }^{5}$ We proceed as follows using an indirect method. The seller can choose $\alpha$ and $\gamma$, which then uniquely determine the cutoff $x^{*}$. Alternatively, if we allow the seller to choose $\gamma$ and $x^{*}$ directly, it is equivalent to allowing the seller to choose $\gamma$ and $\alpha$ indirectly. Therefore, we can rewrite the seller's revenue as a function of $\gamma$ and $x^{*}$ :

$$
\begin{aligned}
R\left(\gamma, x^{*}\right)= & \mu_{H}\left\{\int_{\underline{x}}^{x^{*}} B^{1}(y) g_{H}(y) d y+\int_{x^{*}}^{\bar{x}} B^{2}(y) g_{H}(y) d y\right\} \\
& +\mu_{L}\left\{\int_{\underline{x}}^{x^{*}} B^{1}(y) g_{L}(y) d y+\int_{x^{*}}^{\bar{x}}\left[a+\gamma B^{2}(y)\right] g_{L}(y) d y\right\} \\
& +\mu_{L} \int_{x^{*}}^{\bar{x}} v_{0} g_{L}(y) d y .
\end{aligned}
$$

The following proposition summarizes how return policies affect the seller's revenue

5This can be shown by examining Equations (6), (7), (8), and (9) in Appendix A. 
in this case.

Proposition 5: When $v_{L}+c_{B}>v_{0}$, given $x^{*}$, the seller's revenue is strictly decreasing in $\gamma$, implying that $\gamma=0$ (i.e., no proportional fee) is optimal.

The intuition behind this proposition is as follows. Given the cutoff $x^{*}$, the seller can choose a combination of a fixed fee and a proportional fee consistent with this cutoff. However, using a proportional fee diminishes the seller' revenue since it incentivizes bidders to reduce their bids relative to the fixed-fee case consistent with the same cutoff. This is because higher winning bids imply a higher cost of returning the object in the proportional fee case. In contrast, a fixed fee is a lump sum transfer and does not have this distortion. Therefore, to maximize the seller's revenue, a proportional fee is inferior.

Now we examine the optimal cutoff level of $x^{*}$.

$$
\begin{aligned}
& \frac{\partial R\left(\gamma, x^{*}\right)}{\partial x^{*}} \\
= & \mu_{H} \underbrace{\left[B^{1}\left(x^{*}\right)-B^{2}\left(x^{*}\right)\right]}_{=0} g_{H}\left(x^{*}\right) \frac{d x^{*}}{d \gamma}+\mu_{H} \int_{x^{*}}^{\bar{x}} \frac{\partial B^{2}(y)}{\partial a} \frac{\partial a}{\partial x^{*}} g_{H}(y) d y \\
& +\mu_{L} \int_{x^{*}}^{\bar{x}}\left[1+\gamma \frac{\partial B^{2}(y)}{\partial a}\right] \frac{\partial a}{\partial x^{*}} g_{L}(y) d y+\mu_{L} \underbrace{\left[B^{1}\left(x^{*}\right)-a-\gamma B^{2}\left(x^{*}\right)\right]}_{=v_{L}+c_{B}} g_{L}\left(x^{*}\right) \\
& -\mu_{L} v_{0} g_{L}\left(x^{*}\right) \\
= & \mu_{L}\left(v_{L}+c_{B}-v_{0}\right) g_{L}\left(x^{*}\right) \\
& -\int_{x^{*}}^{\bar{x}} \frac{\mu_{H} \mu_{L}}{\left[\mu_{H} \rho_{1}(y) \rho_{n-1}(y)+\gamma \mu_{L}\right]}\left\{g_{H}(y)-\rho_{1}(y) \rho_{n-1}(y) g_{L}(y)\right\} \frac{\partial a}{\partial x^{*}} d y \\
= & \underbrace{\mu_{L}\left(v_{L}+c_{B}-v_{0}\right) g_{L}\left(x^{*}\right)}_{\text {social welfare effect } \geq 0} \\
& -\underbrace{\text { consumer surplus effect } \geq 0}_{x^{*} \frac{\left(v_{H}-v_{L}\right) \mu_{H} \mu_{L}}{\left[\mu_{H} \rho_{1}\left(x^{*}\right) \rho_{n-1}\left(x^{*}\right)+\gamma \mu_{L}\right]^{2}}\left\{g_{H}(y)-\rho_{1}(y) \rho_{n-1}(y) g_{L}(y)\right\} \frac{d\left[\rho_{1}\left(x^{*}\right) \rho_{n-1}\left(x^{*}\right)\right]}{d x^{*}} d y}
\end{aligned}
$$


In the above expression, either the consumer surplus effect or the social welfare effect could dominate. One observation is that if $v_{H}-v_{L}$ is very small, then the overall sign is positive and it is optimal to induce no return in equilibrium. However, the following example shows that the seller's revenue is not necessarily monotonic in $x *$ in general.

Example 1: Consider two players. Suppose that $v_{0}=0, v_{H}=100+v_{L}$, with $v_{L}$ to be specified later, and $\mu_{H}=\mu_{L}=0.5, c_{B}=0$. We set $\gamma=0$ as this is always optimal for the seller, and examine how the seller's revenue is affected by the return policy by changing $x^{*}$, which then uniquely determines the value of $\alpha$. For $x \in(0,10]$, $F_{H}(x)=\frac{x^{2}}{100}, F_{L}(x)=\frac{x(20-x)}{100}, f_{H}(x)=\frac{x}{50}, f_{L}(x)=\frac{10-x}{50}$. Then $\rho(x)=\frac{f_{H}(x)}{f_{L}(x)}=\frac{x}{10-x}$. Note that $\frac{f_{H}(x)}{f_{L}(x)}$ is indeed strictly increasing as previously assumed. We will vary the value of $v_{L}$ and let it take the values of $0,30,50$ and 80 , respectively.

The results are shown in Figure 1. When $v_{L}=0$, the seller's revenue is decreasing in $x^{*}$; the optimal return policy is $x^{*}=0$, i.e., the full-refund with full-cost-reimbursement policy $\alpha=-c_{B}=0$. When $v_{L}=30$, the seller's revenue first increases, then decreases, and then increases in $x^{*}$; the optimal return policy is a partial-refund policy with $x^{*}=1.2$. When $v_{L}=50$, the seller's revenue first increases, then decreases, and then increases in $\mathrm{x}^{*}$; the optimal return policy is the no-refund policy. When $v_{L}=80$, the seller's revenue is increasing in $x^{*}$; the no refund policy is optimal again. Note that as $v_{L}$ increases, the optimal return policy becomes less generous. This example also 
illustrates the difficulties in determining the condition for an interior optimal return policy as the revenue function is not well behaved.

Figure 1: Plots of Revenue against $x^{*}$
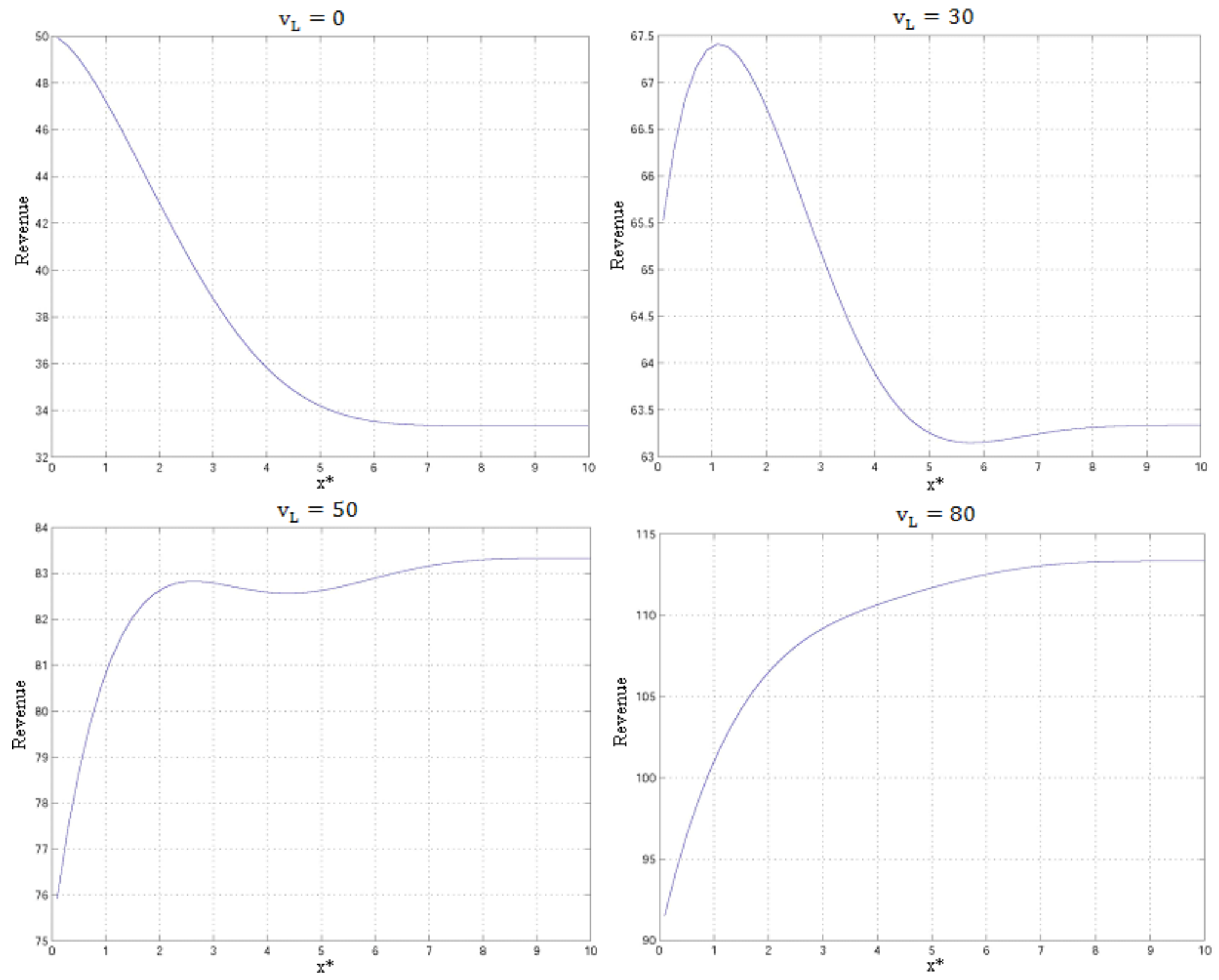


\section{Experimental Design}

Our experiments adopt parameters from Example 1 in the previous section with $v_{L}=0$. We implement second-price auctions with two bidders. ${ }^{6}$ The auctioned item has either the common value $V=100$ or the common value $V=0$ in experimental dollars with equal probability. To make the experiments transparent, the signal generating procedure in practice is a discrete approximation to the continuous distributions in Example 1. In our experiments, the bidder receives a partially informative signal by drawing a numbered chip from an urn containing numbered chips. If $V=100$, the urn contains one 1, two 2's, ..., nine 9's. Alternatively, if $V=0$, the urn contains one 9, two 8 's, ..., nine 1's. The number on the chip is the bidder's signal.

We consider a return policy with a fixed handling fee: if the winning bidder returns the item, s/he gets back the price paid minus the handling fee $\alpha$. Our experimental treatments differ by setting $\alpha$ at four different levels. (1) In the No-Return (NR) treatment, $\alpha=+\infty$, implying that the winning bidder cannot return the item. (2) In the High-Fee (HF) treatment, $\alpha=20$. (3) In the Low-Fee (LF) treatment $\alpha=5$. (4) In the Free-Return (FR) treatment $\alpha=0$.

Proposition 2 and Proposition 4 in section 4 predict that bidders' expected earnings fall while the seller's expected revenues rise as $\alpha$ decreases toward zero. However, when $\alpha=0$, there are multiple equilibria. One equilibrium involves all bidders bidding 100

${ }^{6}$ We used two bidders in each auction since as the number of bidders increases, the bidding function becomes flatter, making it more difficult to test the impact of signals on bids. 
with the winner returning the item when $V=0$. This equilibrium is efficient, creating the maximum possible surplus. If weakly dominated strategies are allowed, there are other inefficient equilibria that involve bids above 100 with the winner returning the item regardless of whether $V=0$ or $V=100$. These equilibria have very different implications for a seller selecting a free-return policy. In the case of the efficient equilibrium, the seller extracts the maximum possible revenue from the bidder. However, in the case of the inefficient equilibria, the seller receives no revenue at all. One goal of this study is to test empirically which equilibrium arises when $\alpha=0$.

Treatments were implemented in two-day sequences of one-hour sessions. On day one of the two-day sequence, the recruited participants first participated in two rounds of practice auctions. The first round of practice auctions was hand-run and real urns with numbered chips were presented to participants. Starting from the second practice round and throughout the rest of the session, the auctions and the signal-generating procedure were computerized in a manner analogous to the hand-run method used during the first practice round. After the practice rounds, the participants began the 15 monetary-payoff rounds with 225 experimental dollars of capital endowment. In each round, participants were randomly and anonymously matched into markets of two bidders. Participants were informed that if their net balance dropped to zero or below, they would no longer be permitted to continue playing. ${ }^{7}$ Day two of the two-day sequence took place one week

\footnotetext{
${ }^{7}$ No participant went bankrupt in any of our experiments.
} 
later, and the same participants were invited back. On day two, procedures were the same as on day one except that there was no hand-run practice round. To give participants an incentive to return on day two, their earnings on day one were retained until the completion of the day-two session.

There were four two-day sequences for each of the NR, HF, LF and FR treatments. No participant was allowed to participate in more than one two-day sequence. There were 8-12 participants in each sequence. Table 1 presents details on the number of participants on days one and two of each sequence.

Table 1: Number of Participants in Each Two-Day Sequence

\begin{tabular}{|c|c|c|c|c|c|c|c|c|c|c|c|}
\hline NR & Day 1 & Day 2 & $\mathrm{HF}$ & Day 1 & Day 2 & LF & Day 1 & Day 2 & FR & Day 1 & Day 2 \\
\hline 1 & 12 & 12 & \multirow{4}{*}{$\begin{array}{l}1 \\
2 \\
3 \\
4\end{array}$} & 10 & 10 & \multirow{4}{*}{$\begin{array}{l}1 \\
2 \\
3 \\
4\end{array}$} & 12 & 12 & \multirow{4}{*}{$\begin{array}{l}1 \\
2 \\
3 \\
4\end{array}$} & 12 & 10 \\
\hline 2 & 12 & 10 & & 10 & 8 & & 12 & 12 & & 12 & 10 \\
\hline 3 & 12 & 8 & & 12 & 12 & & 12 & 12 & & 12 & 12 \\
\hline 4 & 10 & 8 & & 12 & 10 & & 12 & 12 & & 12 & 10 \\
\hline
\end{tabular}

We conducted our experiments at the Experimental Economics Laboratory, Shanghai University of Finance and Economics (SUFE). The participants were recruited from a campus-wide list of undergraduate students who had previously responded to an announcement in a campus-wide required first-year undergraduate course. None of the participants had any experience with common-value auction experiments. All laboratory sessions were computerized using Visual Basic 6.0. Both the instructions and the information shown on the computer screen were in Chinese. The average payment was 44.07 RMB (15 experimental dollars were equivalent to $1 \mathrm{RMB}$ and the exchange rate 
was US\$1 $=6.23 \mathrm{RMB}$ ) for the two one-hour sessions making up a two-day sequence.

Since the average hourly wage in Shanghai for a college graduate is about 15-20 RMB,

44.07 RMB is a considerable amount for undergraduate students.

\section{Results}

This section reports experimental results in the monetary-payoff rounds.

\subsection{Bids}

Figure 2 shows the bidders' average bids for each treatment, conditional on the signal received. Figure 2 suggests that a more generous return policy is associated with higher bids as predicted by theory.

Figure 2: Treatment Difference in Bids

\section{Treatment Difference: Inexperienced}

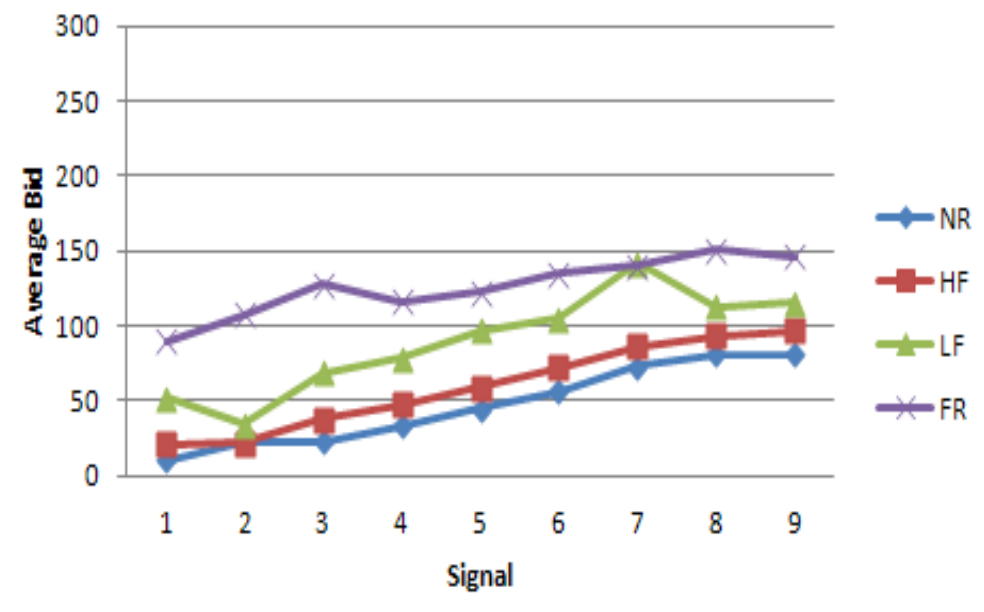

\section{TreatmentDifference: Experienced}

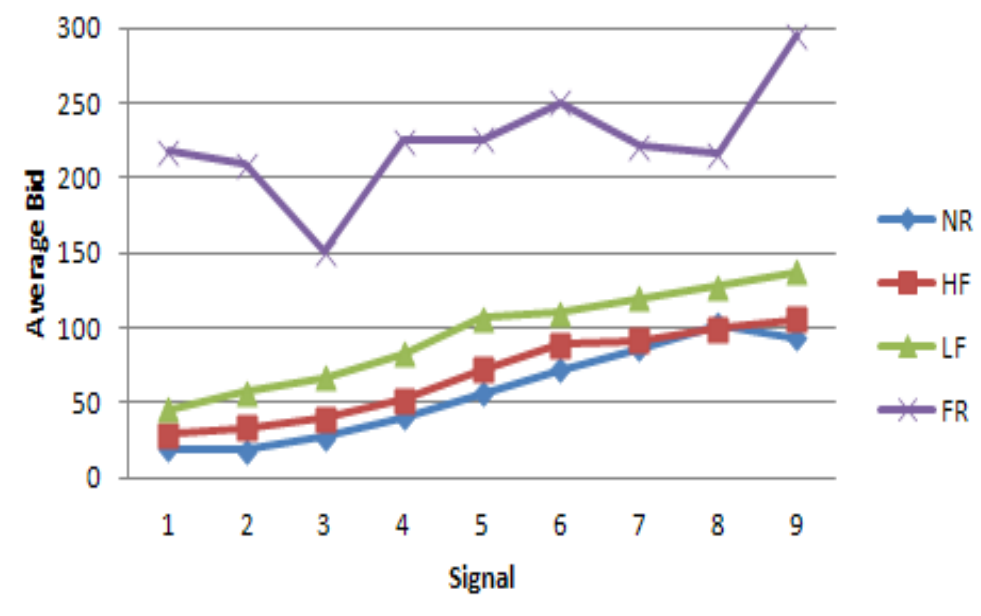

Treating each session's average bid as one independent observation, the

Wilcoxon-Mann-Whitney rank-sum tests show that for inexperienced bidders (sessions 
on day 1), bids in the NR treatment were significantly lower than those in the HF treatment $(p=0.043)$, while bids in the HF treatment were significantly lower than those in the LF treatment $(p=0.043)$. However, the difference in bids was not significant between the LF and FR treatments $(p=0.149)$. For experienced bidders (sessions on day 2), the pattern was similar, but not identical: while bids in the NR treatment were not significantly different from those in the HF treatment $(p=0.248)$, bids in the HF treatment were significantly lower than those in the LF treatment $(p=0.021)$, and bids in the LF treatment were lower than in the FR treatment with marginal significance $(p=0.083)$.

Next we examine whether experienced bidders bid closer than inexperienced bidders to the theoretically predicted bids. For the multiple-equilibrium FR treatment, we use the efficient equilibrium in which all bidders bid 100 regardless of the signal received as our theoretical benchmark. Figure 3 suggests that experience does not help bring bids closer to the theoretical prediction in any of the four treatments. The mean squared deviation (MSD) between the actual bids in the experimental market and the value predicted by the model is measured as follows:

$$
\operatorname{MSD}\left[b, b^{*}(x)\right]=\frac{1}{T} \sum_{i=1}^{T}\left(b-b^{*}(x)\right)^{2}
$$

where $b$ is the actual bid, $b^{*}(x)$ is the predicted bid conditional on the signal $x$ received by the bidder, and $T$ is the total number of bids in the monetary-payoff rounds within a 
session. Comparing the MSD in the inexperienced sessions with the MSD in the experienced sessions, the Wilcoxon signed-ranks test yields no significant difference in any of the four treatments $(p=0.715$ in NR, $p=1.000$ in HF, $p=0.715$ in LF and $p=0.144$ in FR). Moreover, in the FR treatment, many bidders bid higher than 100, behavior consistent with the equilibria involving weakly dominated strategies.

Figure 3: Actual Bids vs. Predictions
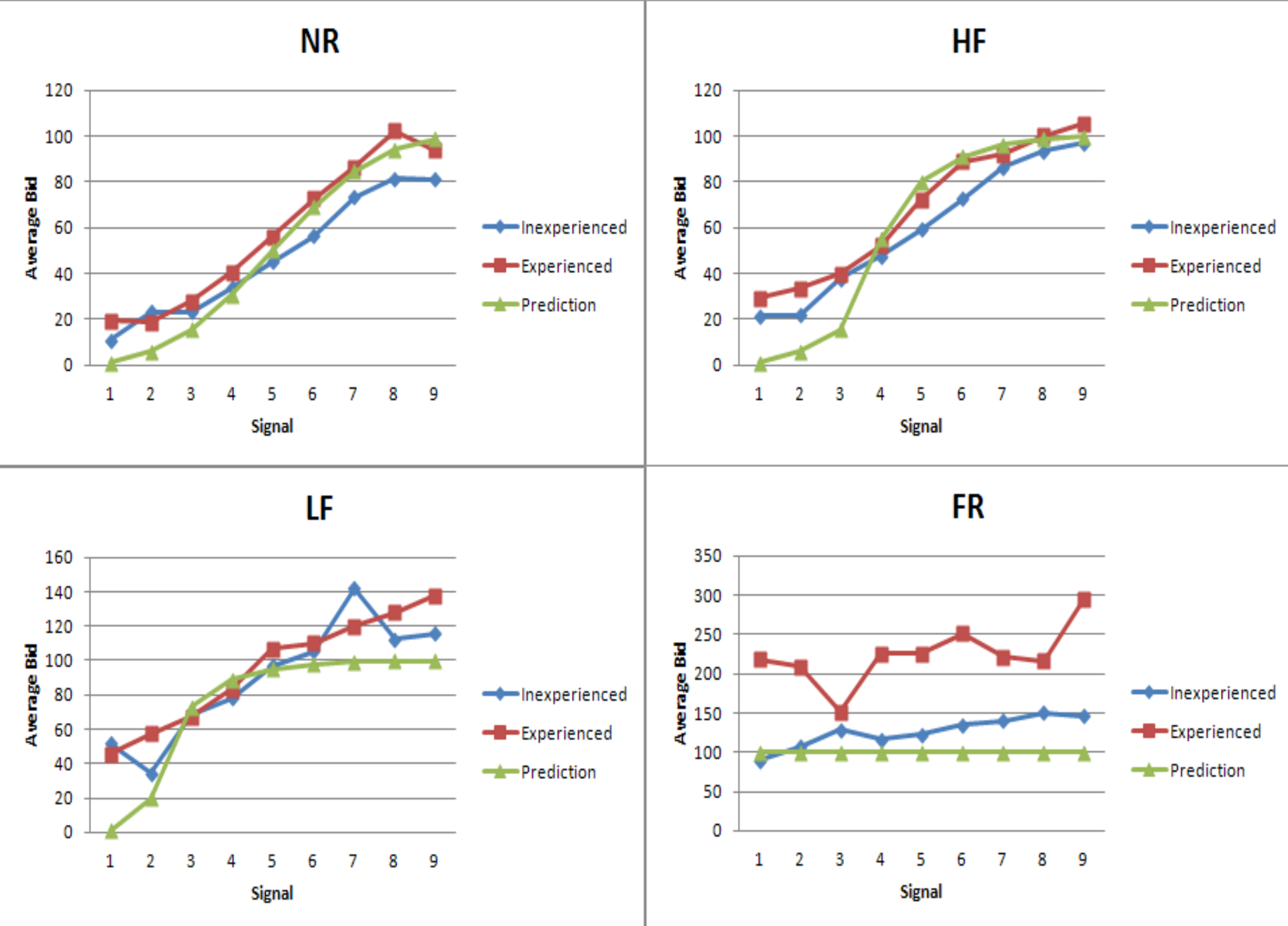
In Table 2 we report a random-effects regression (with random effects both at the session level and at the individual level) for the determinants of bids over all monetary-payoff rounds. The regression shows that in the NR treatment bids increased significantly as the signal increased (the coefficient for Signal was positive with $p=0.000)$. In both the LF and HF treatments, the relationship between bids and signals was close to the NR relationship (neither the coefficient of Signal*LF nor that of Signal*HF was significantly different from zero with $p=0.368$ and $p=0.995$, respectively). However, in the FR treatment, the empirical bidding function was much flatter than in the NR treatment (the coefficient of Signal*FR is negative with $p=$ 0.000 ), though the relationship between bids and signals was still positive (the Wald $\chi^{2}$ test shows that the sum of the coefficients of Signal and Signal*FR is positive with $p=0.000)$. The regression also indicates that bidders tended to bid higher during the later rounds of the FR treatment (the coefficient of Round*FR is positive with $p=$ 0.000), while round had no significant impact on bids in the other treatments. The empirical bidding function for the FR treatment reflects the fact that many bidders placed bids greater than 100 regardless of the signal received. Such behavior creates no surplus for either the bidder or the seller. In this treatment, bidders generally earned nothing since, as predicted by theory, the seller captured any surplus created by trade. We conjecture that as the experiment proceeded the fun of winning the auctioned item and then returning it began to dominate concern with monetary payoffs, which were always zero in 
any case. Thus, bids rose as bidders competed to win (and then return) the item. As mentioned previously, bidding above 100 is consistent with the inefficient equilibria involving weakly dominated strategies.

Table 2: Determinants of Bids

\begin{tabular}{c|c|c}
\hline & Coef. & Std. Err. \\
\hline Constant & -9.737 & 26.11 \\
FR dummy & 53.847 & 36.81 \\
LF dummy & 37.272 & 36.77 \\
HF dummy & 9.333 & 37.07 \\
Signal & $10.612^{* * *}$ & 0.77 \\
Signal*FR & $-4.575^{* * *}$ & 1.06 \\
Signal*LF & 0.935 & 1.04 \\
Signal*HF & -0.007 & 1.08 \\
Experience dummy & 10.151 & 36.75 \\
ExperienceFR & $88.613^{*}$ & 51.62 \\
Experience*LF & -4.050 & 51.32 \\
Experience*HF & -2.053 & 51.94 \\
Round & 0.510 & 0.44 \\
Round*FR & $5.984 * * *$ & 0.61 \\
Round*LF & -0.065 & 0.60 \\
Round*HF & 0.383 & 0.62 \\
\hline Obs. & \multicolumn{2}{|c}{5310} \\
Wald Chi2 & \multicolumn{2}{|c}{-30467.167} \\
Log likelihood & \multicolumn{2}{|c}{} \\
\hline
\end{tabular}

* indicates significance at $p=0.10$ (two-tailed tests); *** indicate significance at $p=$ 0.01 (two-tailed tests).

\subsection{Earnings}

Proposition 2 in section 4.1 predicts that consumer surplus is lower with a more generous return policy. Figure 4 suggests that this is true empirically. A bidder's total 
payoff (in experimental currency) in a session decreases as the handling fee for returning the auctioned item goes down. Compared with payoffs to inexperienced bidders on day 1 , the average total payoffs for experienced bidders on day 2 were closer to the theoretical prediction (i.e., the ex ante expected total payoff in 15 monetary-payoff rounds plus 225).

Figure 4: Average Total Payoff

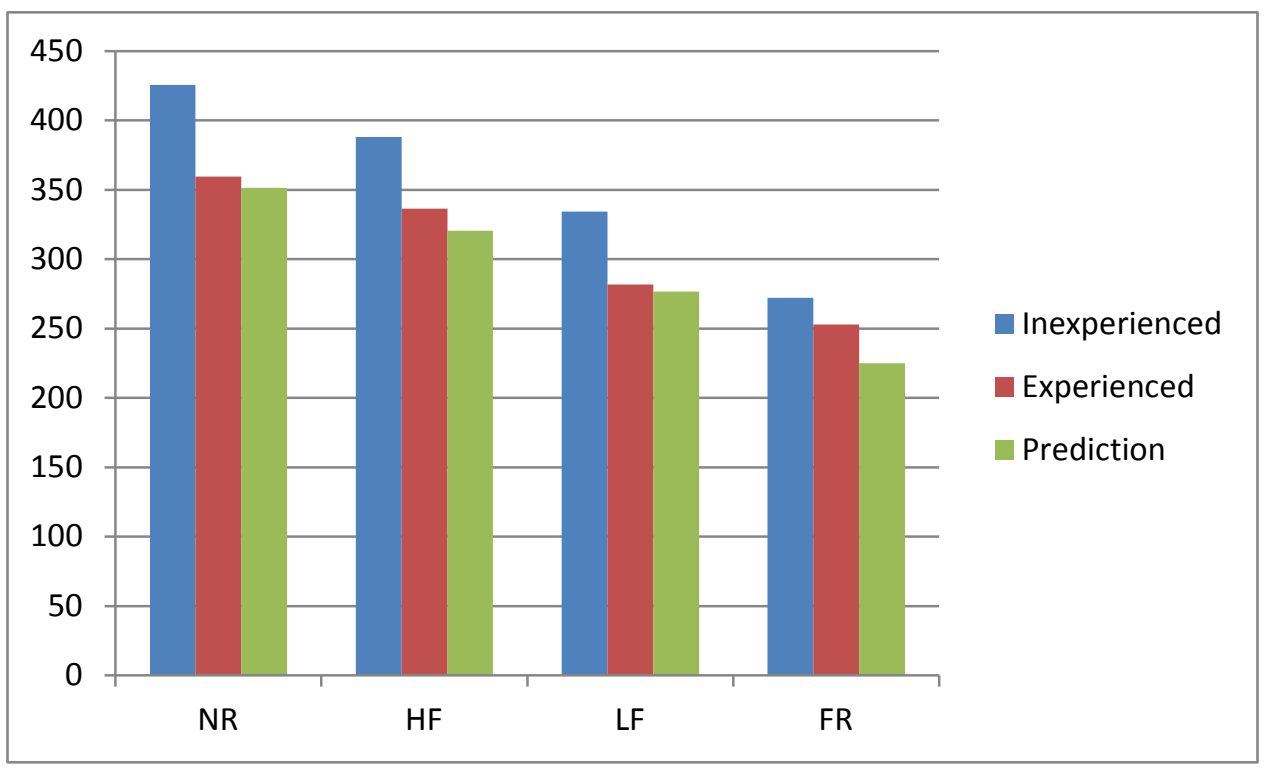

Treating each session's average total payoff as one independent observation, the Wilcoxon-Mann-Whitney rank-sum tests show that for inexperienced bidders (sessions on day 1), while total payoffs in the NR treatment were not significantly different from those in the HF treatment $(p=0.248)$, total payoffs in the HF treatment were significantly higher than in the LF treatment $(p=0.083)$ and total payoffs in the LF treatment were also significantly higher than in the FR treatment $(p=0.043)$. For 
experienced bidders (sessions on day 2), the pattern is similar: while total payoffs in the NR treatment were not significantly different from those in the HF treatment $(p=0.387)$, total payoffs in the HF treatment were significantly higher than those in the LF treatment $(p=0.043)$ and total payoffs in the LF treatment were also significantly higher than in the FR treatment $(p=0.021)$.

A random-effects regression (with random effects both at the session and at the individual level) for treatment differences in bidders' payoffs in each auction in Table 3 confirms the observation in Figure 4. Bidders' payoffs are higher in the NR treatment, compared with the FR and the LF (the signs of the coefficients of the FR and LF dummies are significantly negative). Compared with day 1 , bidders earn less on day 2 when they have more experience but simultaneously bid against more experienced opponents (the sign of the experience dummy is significantly negative). A Wald $\chi^{2}$ tests indicate that the coefficient of the FR dummy is significantly lower than that of the LF dummy $(p=0.009)$, and the coefficient of LF is significantly lower than that of HF $(p=0.026)$. The Wald $\chi^{2}$ test also shows that we cannot reject the joint hypothesis that all three interactions of treatment dummies with experience equal zero $(p=0.608)$. This confirms that the treatment effects hold both with and without experience. 
Table 3: Comparing Bidders' Payoffs

\begin{tabular}{c|c|c}
\hline & Coef. & Std. Err. \\
\hline Constant & $13.351^{* * *}$ & 1.15 \\
FR dummy & $-10.218^{* * *}$ & 1.61 \\
LF dummy & $-6.069^{* * *}$ & 1.61 \\
HF dummy & -2.459 & 1.64 \\
Experience dummy & $-4.304^{* *}$ & 1.69 \\
Experience*FR & 3.024 & 2.35 \\
Experience*LF & 0.803 & 2.32 \\
Experience*HF & 0.931 & 2.38 \\
\hline Obs. & \multicolumn{2}{|c}{} \\
Wald Chi2 & 5310 \\
\hline
\end{tabular}

$* *$ indicates significance at $p=0.05$ (two-tailed tests); $* * *$ indicates significance at $p=$ 0.01 (two-tailed tests).

Figure 5 examines the efficiency loss associated with return policies. Setting the seller's value for the auctioned item at zero, there is a loss in aggregate surplus if the winner of the auction chooses to return the item when $V=100$. In the HF and LF treatments, winners rarely return the item when $V=100$. However, in the FR treatment, the frequency of returning the item when $V=100$ is 0.266 for inexperienced bidders, and 0.497 for experienced bidders. We observe significant efficiency loss associated with the FR treatment. To compare the frequency of returning the high quality $\mathrm{V}=100$ items across treatments, we run Wilcoxon-Mann-Whitney rank-sum tests with the following results: $\mathrm{HF}<\mathrm{LF}(p=0.047), \mathrm{LF}<\mathrm{FR}(p=0.021)$ for inexperienced bidders; $\mathrm{HF}=\mathrm{LF}(p=0.850), \mathrm{LF}<\mathrm{FR}(p=0.018)$ for experienced bidders (treating the session average return frequency as one independent observation). 
Figure 5: Choice of Return when $V=100$ (in Percentage)

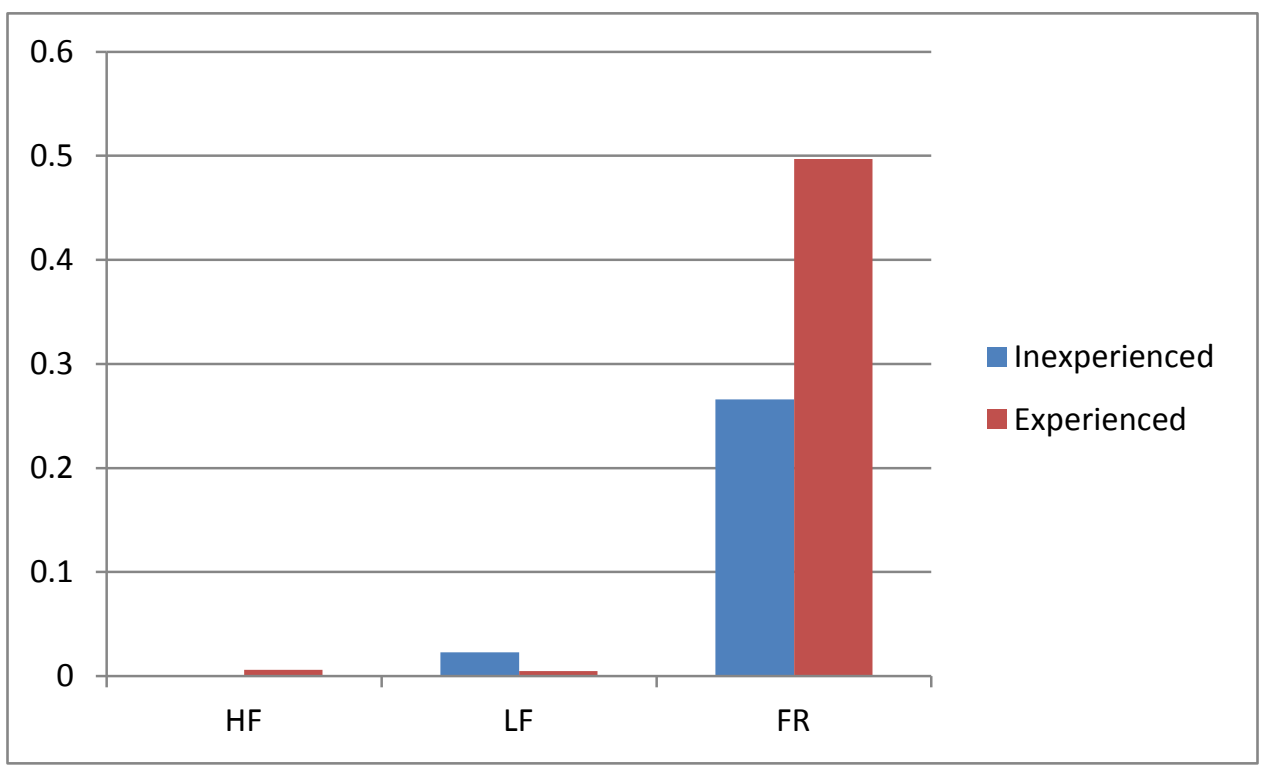

Proposition 4 in section 4.2, predicted that given our experimental parameters (in particular $v_{L}=c_{B}=0$ ) if the seller's value of the item is zero, seller revenue should increase with the generosity of the return policy and the free return policy should be optimal for the sellers. However, this proposition was derived under the assumption that the efficient equilibrium would prevail in the FR case. Figure 6 compares the bidders' payments to the sellers across treatments. In general, the bidders' average payment to the sellers increases as the handling fee for returning the auctioned item decreases as predicted. However, the payments are not highest in the FR treatment because many winning bids exceed 100 with the winners choosing to return the item when $V=100$. Thus, the efficient equilibrium did not prevail in the FR case as was assumed in the theoretical derivation, and this was detrimental to seller revenues. The payments are 
actually highest in the LF rather than in the FR treatment.

Figure 6: Bidders' Average Payment to the Sellers

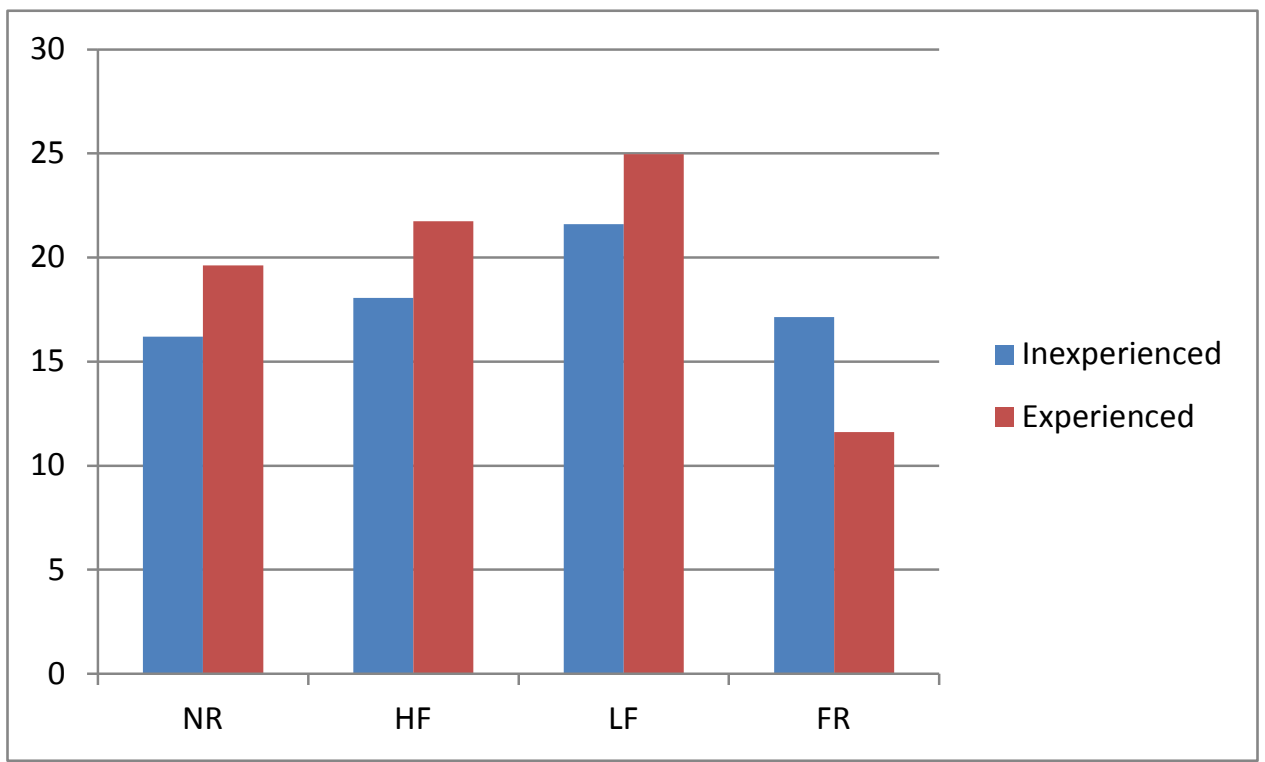

Treating the average payment in each session as one independent observation, Wilcoxon-Mann-Whitney rank-sum tests yield the following results: NR $=\mathrm{HF}$ $(p=0.564), \mathrm{HF}<\mathrm{LF}(p=0.083), \mathrm{LF}>\mathrm{FR}(p=0.083), \mathrm{LF}>\mathrm{NR}(p=0.043)$ for inexperienced bidders; $\mathrm{NR}=\mathrm{HF} \quad(p=0.248), \quad \mathrm{HF}<\mathrm{LF} \quad(p=0.043), \quad \mathrm{LF}>\mathrm{FR}$ $(p=0.021), \mathrm{LF}>\mathrm{NR}(p=0.021)$ for experienced bidders.

A random-effects regression (with random effects both at the session and at the individual level) for treatment differences in bidders' payments to sellers in each auction in Table 4 confirms this observation. Notice that in the FR treatment, bidders transfer less money to the sellers in the day-2-sessions compared with the day-1-sessions (the 
coefficient of Experience*FR is negative with $p=0.014)$. We conjecture that this is because after experiencing low payoffs during the day-1-sessions, the fun of winning becomes paramount, driving more bidders to bid above 100 and subsequently return the auctioned item for a full refund in the day-2-sessions.

Table 4: Comparing Bidders' Payments to Sellers

\begin{tabular}{c|c|c}
\hline & Coef. & Std. Err. \\
\hline Constant & $16.199^{* * *}$ & 1.76 \\
FR dummy & 0.946 & 2.46 \\
LF dummy & $5.408^{* *}$ & 2.46 \\
HF dummy & 1.868 & 2.51 \\
Experience dummy & 3.423 & 2.61 \\
Experience*FR & $-8.942^{* *}$ & 3.63 \\
Experience*LF & -0.060 & 3.57 \\
Experience*HF & 0.252 & 3.69 \\
\hline Obs. & \multicolumn{2}{|c}{5310} \\
Wald Chi2 & 36.55 \\
\hline
\end{tabular}

** indicates significance at $p=0.05$ (two-tailed tests); $* * *$ indicates significance at $p=$ 0.01 (two-tailed tests).

\section{Conclusions}

This paper investigates the role of linear return policies in second-price auctions. The equilibrium is unique unless returns are free. With a more generous return policy, bidders act more aggressively. Since the winning bidder pays more, the consumer surplus is lower in such auctions. For sellers, we demonstrate that a revenue-maximizing seller should never use a return fee that is proportional to the price paid for an item. Rather a fixed return fee should be used. Furthermore, since the winning bidder may return the 
object when s/he obtains more information regarding its value, a higher bid induced by a more generous return policy, while hurting bidders, may not always be beneficial to the seller. Only when the efficiency losses from returns are relatively small will a more generous return policy help the seller.

Our laboratory observations support the theoretical prediction that the seller's revenue increases as the handling fee for returning the auctioned item decreases, but remains positive. When returning the item is free, many bidders bid above the highest possible value and subsequently return the item regardless of the revealed value. While this is consistent with equilibrium behavior, it is an inefficient equilibrium that is not optimal for the seller.

In theory, there exist optimal mechanisms for sellers to maximize revenue. For our case of common values, a seller can extract the full surplus from bidders. ${ }^{8}$ However, those optimal mechanisms are not commonly observed in reality, partly because too much detail regarding the underlining environment is required for the seller to design an optimal mechanism. The discrepancy between theory and common practice prompts the claim that a set of simplicity and robustness criteria should be imposed on the trading mechanisms. ${ }^{9}$ Our auctions with return policies are the sort of simple and familiar

\footnotetext{
8See Cremer and Mclean [4] and McAfee and Reny [17].

${ }^{9}$ Hurwicz [12] illustrates the need for mechanisms that are independent of the parameters of the model. Wilson [20] points out that a desirable property of a trading rule is that it "does not rely on features of the agents". Lopomo [15] [16] requires mechanisms to exhibit "simplicity" and " robustness".
} 
trading procedures that Hurwicz [12], Lopomo [15] [16], and Wilson [20] advocate.

Furthermore, as we have shown in this paper, return policies, while being "simple" instruments, can be effective at increasing seller revenue under certain circumstances.

\section{References}

[1] Abhishek, V., Hajek, B. E., and Williams, S. R. Common value auctions with a profit sharing contract. CoRR abs/1102.3195 (2011).

[2] Casari, M., Ham, J., and Kagel, J. (2007). Selection bias, demographic effects, and ability effects in common value auction experiments. The American Economic Review, 97, 4 1278-1304.

[3] Che, Y.-K. Customer return policies for experience goods. Journal of Industrial Economics 44, 1 (March 1996), 17-24.

[4] Cremer, J., and McLean, R. P. Full extraction of the surplus in Bayesian and dominant strategy auctions. Econometrica 56, 6 (November 1988), 1247-1257.

[5] DeMarzo, P. M., Kremer, I., and Skrzypacz, A. Bidding with securities: Auctions and security design. American Economic Review 95, 4 (September 2005), 936-959.

[6] Garvin, S., and Kagel, J. (1994). Learning in common value auctions: Some initial observations. Journal of Economic Behavior \& Organization, 25, 351-371.

[7] Goertz, J. M. M. (2012) Market composition and experience in common-value auctions. Experimental Economics 15, 106-127.

[8] Hafalir, I., and Yektas, H. Selling goods of unknown quality: forward versus spot auctions. Review of Economic Design 15, 3 (September 2011), 245-256.

[9] Haile, P. A. Auctions with private uncertainty and resale opportunities. Journal of Economic Theory 108, 1 (January 2003), 72-110.

[10] Hansen, R. G. Auctions with contingent payments. American Economic Review 75, 4 (September 1985), 862-865.

[11] Huang, Z., Qiu, Y., and Matsubara, S. Designing a refundable auction for limited capacity suppliers. Third International Conference on Semantics, Knowledge and Grid 
(2007), 104-109.

[12] Hurwicz, L. On informationally decentralized systems. In Decision and Organization, ed. by R. Radner and C. McGuire (1972), Amsterdam: North-Holland, 297-336.

[13] Kagel, J., and Levin, D. (1986). The winner's curse and public information in common value auctions. The American Economic Review, 76, 5, 894-920.

[14] Kagel, J., and Levin, D. (2002). Bidding in common value auctions: A survey of experimental research. In J. Kagel and D. Levin (Eds.), Common value auctions and the winner's curse. Princeton: Princeton University Press, 1-84.

[15] Lopomo, G. The English auction is optimal among simple sequential auctions. Journal of Economic Theory 82, 1 (1998), 144-166.

[16] Lopomo, G. Optimality and robustness of the English auction. Games and Economic Behavior 36, 2 (August 2001), 219-240.

[17] McAfee, R. P., and Reny, P. J. Correlated information and mechanism design. Econometrica 60, 2 (March 1992), 395-421.

[18] Milgrom, P. R., and Weber, R. J. A theory of auctions and competitive bidding. Econometrica 50, 5 (September 1982), 1089-1122.

[19] Riley, J. G. Ex post information in auctions. Review of Economic Studies 55, 3 (July 1988), 409-29.

[20] Wilson, R. Competitive bidding with disparate information. Management Science 15, 7 (March 1969), 446-448.

[21] Zhang, J. Revenue maximizing with return policy when buyers have uncertain valuations. International Journal of Industrial Organization 31, 5 (2013), 452-461. 


\section{Appendix A: Proofs}

We will make use of the following lemma repeatedly in our analysis. The proof is standard and is thus omitted.

Lemma 1 Suppose that $F_{H}$ dominates $F_{L}$ in likelihood ratio, i.e., $\rho_{1}(x)$ is increasing in $x$. Then

1. $F_{H}$ dominates $F_{L}$ in hazard rate, i.e. $\frac{f_{H}(x)}{1-F_{H}(x)} \leq \frac{f_{L}(x)}{1-F_{L}(x)}, \forall x$.

2. $F_{H}$ dominates $F_{L}$ in reversed hazard rate, i.e. $\frac{f_{H}(x)}{F_{H}(x)} \geq \frac{f_{L}(x)}{F_{L}(x)}, \forall x$.

3. $\frac{F_{H}(x)}{F_{L}(x)}$ is increasing in $x$.

4. $\rho_{k}(x)$ is increasing in $x, \forall k$.

This lemma also implies another property of our likelihood ratio dominance assumption.

Lemma 2 Suppose that $F_{H}$ dominates $F_{L}$ in likelihood ratio. Then

$$
g_{H}(x)-g_{L}(x) \rho_{1}(x) \rho_{n-1}(x) \geq 0, \quad \forall x .
$$

\section{Proof for Lemma 2}

$$
\begin{aligned}
& g_{H}(x)-g_{L}(x) \rho_{1}(x) \rho_{n-1}(x) \\
= & g_{L}(x)\left[\frac{g_{H}(x)}{g_{L}(x)}-\rho_{1}(x) \rho_{n-1}(x)\right] \\
= & g_{L}(x)\left\{\frac{n(n-1) f_{H}(x) F_{H}(x)^{n-2}\left[1-F_{H}(x)\right]}{n(n-1) f_{L}(x) F_{L}(x)^{n-2}\left[1-F_{L}(x)\right]}-\frac{f_{H}(x)}{f_{L}(x)} \frac{f_{H}(x) F_{H}(x)^{n-2}}{f_{L}(x) F_{L}(x)^{n-2}}\right\} \\
= & g_{L}(x) \frac{f_{H}(x) F_{H}(x)^{n-2}\left[1-F_{H}(x)\right]}{f_{L}(x)^{2} F_{L}(x)^{n-2}}\left[\frac{f_{L}(x)}{1-F_{L}(x)}-\frac{f_{H}(x)}{1-F_{H}(x)}\right] \\
\geq & 0 .
\end{aligned}
$$

The above inequality follows directly from Lemma 1. 


\section{Proof for Proposition 2}

We first consider the effect of the fixed fee $a$.

$$
\begin{aligned}
& \frac{\partial C S(a, \gamma)}{\partial a} \\
= & \mu_{H} \underbrace{\left\{\left[v_{\mathrm{H}}-B^{1}\left(x^{*}\right)\right]-\left[v_{H}-B^{2}\left(x^{*}\right)\right]\right\}}_{=0} g_{H}\left(x^{*}\right) \frac{d x^{*}}{d a}-\mu_{H} \int_{x^{*}}^{\bar{x}} \frac{\partial B^{2}(y)}{\partial a} g_{H}(y) d y \\
& -\mu_{L} \int_{x^{*}}^{\bar{x}} \frac{\left.\partial a+\gamma B^{2}(y)\right]}{\partial a} g_{L}(y) d y+\mu_{L} \underbrace{\left[\left[v_{L}-B^{1}\left(x^{*}\right)\right]+a+\gamma B^{2}\left(x^{*}\right)+c_{B}\right]}_{=0} g_{L}\left(x^{*}\right) \frac{d x^{*}}{d a} \\
= & -\mu_{H} \int_{x^{*}}^{\bar{x}} \frac{-\mu_{L}}{\mu_{H} \rho_{1}(y) \rho_{n-1}(y)+\gamma \mu_{L}} g_{H}(y) d y-\mu_{L} \int_{x^{*}}^{\bar{x}} \frac{\mu_{H} \rho_{1}(y) \rho_{n-1}(y) g_{L}(y)}{\mu_{H}(y) \rho_{n-1}(y)+\gamma \mu_{L}} d y \\
= & \int_{x^{*}}^{\bar{x}} \frac{\mu_{L} \mu_{H}}{\mu_{H} \rho_{1}(y) \rho_{n-1}(y)+\gamma \mu_{L}}\left\{g_{H}(y)-\rho_{1}(y) \rho_{n-1}(y) g_{L}(y)\right\} d y
\end{aligned}
$$

Therefore, according to Lemma 2 , the consumer surplus is increasing in $a$.

Now consider the effect of the percentage fee $\gamma$.

$$
\begin{aligned}
& \frac{\partial C S(a, \gamma)}{\partial \gamma} \\
= & \mu_{H} \underbrace{\left\{\left[v_{H}-B^{1}\left(x^{*}\right)\right]-\left[v_{H}-B^{2}\left(x^{*}\right)\right]\right\}}_{=0} g_{H}\left(x^{*}\right) \frac{d x^{*}}{d \gamma} \\
& -\mu_{H} \int_{x^{*}}^{\bar{x}} \frac{\partial B^{2}(y)}{\partial \gamma} g_{H}(y) d y-\mu_{L} \int_{x^{*}}^{\bar{x}} \frac{\left.\partial \gamma B^{2}(y)\right]}{\partial \gamma} g_{L}(y) d y \\
& +\mu_{L} \underbrace{\left[\left[v_{L}-B^{1}\left(x^{*}\right)\right]+a+\gamma B^{2}\left(x^{*}\right)+c_{B}\right]}_{=0} g_{L}\left(x^{*}\right) \frac{d x^{*}}{d \gamma} \\
= & -\mu_{H} \int_{x^{*}}^{\bar{x}} \frac{-\mu_{L}\left[\mu_{H} v_{H} \rho_{1}(y) \rho_{n-1}(y)-\left(a+c_{B}\right) \mu_{L}\right]}{\left[\mu_{H} \rho_{1}(y) \rho_{n-1}(y)+\gamma \mu_{L}\right]^{2}} g_{H}(y) d y \\
& -\mu_{L} \int_{x^{*}}^{\bar{x}} \frac{\mu_{H} \rho_{1}(y) \rho_{n-1}(y)\left[\mu_{H} v_{H} \rho_{1}(y) \rho_{n-1}(y)-\left(a+c_{B}\right) \mu_{L}\right]}{\left[\mu_{H} \rho_{1}(y) \rho_{n-1}(y)+\gamma \mu_{L}\right]^{2}} g_{L}(y) d y \\
= & \int_{x^{*}}^{\bar{x}} \frac{\mu_{H} \mu_{L}\left[\mu_{H} v_{H} \rho_{1}(y) \rho_{n-1}(y)-\left(a+c_{B}\right) \mu_{L}\right]}{\left[\mu_{H} \rho_{1}(y) \rho_{n-1}(y)+\gamma \mu_{L}\right]^{2}}\left\{g_{H}(y)-\rho_{1}(y) \rho_{n-1}(y) g_{L}(y)\right\} d y
\end{aligned}
$$

Therefore, the consumer surplus is increasing in $\gamma$.

\section{Proof for Proposition 3}

$$
\begin{aligned}
& \frac{\partial W(a, \gamma)}{\partial a}=\mu_{L}\left(v_{L}+c_{B}-v_{0}\right) \frac{\partial x^{*}}{\partial a} g_{L}\left(x^{*}\right), \\
& \frac{\partial W(a, \gamma)}{\partial \gamma}=\mu_{L}\left(v_{L}+c_{B}-v_{0}\right) \frac{\partial x^{*}}{\partial \gamma} g_{L}\left(x^{*}\right) .
\end{aligned}
$$


As a result, the social welfare is increasing in $a$ and $\gamma$ if and only if $v_{L}+c_{B} \geq v_{0}$.

\section{Proof for Proposition 5}

We first examine how $\gamma$ affects the revenue.

$$
\begin{aligned}
& \frac{\partial R\left(\gamma, x^{*}\right)}{\partial \gamma} \\
& =\mu_{H} \int_{x^{*}}^{\bar{x}}\left[\frac{\partial B^{2}(y)}{\partial \gamma}+\frac{\partial B^{2}(y)}{\partial a} \frac{\partial a}{\partial \gamma}\right] g_{H}(y) \\
& +\mu_{L} \int_{x^{*}}^{\bar{x}}\left\{\frac{\partial a}{\partial \gamma}+\frac{\left.\partial \gamma B^{2}(y)\right]}{\partial \gamma}+\gamma \frac{\partial B^{2}(y)}{\partial a} \frac{\partial a}{\partial \gamma}\right\} g_{L}(y) d y \\
& =\int_{x^{*}}^{\bar{x}}\left[\mu_{H} \frac{\partial B^{2}(y)}{\partial \gamma} g_{H}(y)+\mu_{L} \frac{\left.\partial \gamma B^{2}(y)\right]}{\partial \gamma} g_{L}(y)\right] d y \\
& +\int_{x^{*}}^{\bar{x}}\left\{\mu_{H} \frac{\partial B^{2}(y)}{\partial a} g_{H}(y)+\mu_{L}\left[1+\gamma \frac{\partial B^{2}(y)}{\partial a}\right] g_{L}(y)\right\} \frac{\partial a}{\partial \gamma} d y \\
& =\int_{x^{*}}^{\bar{x}}\left[\mu_{H} \frac{-\mu_{L}\left[\mu_{H} v_{H} \rho_{1}(x) \rho_{n-1}(x)-\mu_{L}\left(a+c_{B}\right)\right]}{\left[\mu_{H} \rho_{1}(x) \rho_{n-1}(x)+\gamma \mu_{L}\right]^{2}} g_{H}(y)\right. \\
& \left.+\mu_{L} \frac{\mu_{H} \rho_{1}(x) \rho_{n-1}(x)\left[\mu_{H} v_{H} \rho_{1}(x) \rho_{n-1}(x)-\mu_{L}\left(a+c_{B}\right)\right]}{\left[\mu_{H} \rho_{1}(x) \rho_{n-1}(x)+\gamma \mu_{L}\right]^{2}} g_{L}(y)\right] d y \\
& +\int_{x^{*}}^{\bar{x}}\left\{\mu_{H} \frac{-\mu_{L}}{\mu_{H} \rho_{1}(x) \rho_{n-1}(x)+\gamma \mu_{L}} g_{H}(y)\right. \\
& \left.+\mu_{L} \frac{\mu_{H} \rho_{1}(x) \rho_{n-1}(x)}{\mu_{H} \rho_{1}(x) \rho_{n-1}(x)+\gamma \mu_{L}} g_{L}(y)\right\} \frac{\partial a}{\partial \gamma} d y \\
& =\int_{x^{*}}^{\bar{x}} \frac{\mu_{L} \mu_{H}\left[\mu_{H} v_{H} \rho_{1}(x) \rho_{n-1}(x)-\mu_{L}\left(a+c_{B}\right)\right]}{\left[\mu_{H} \rho_{1}(y) \rho_{n-1}(y)+\gamma \mu_{L}\right]^{2}}\left\{\rho_{1}(y) \rho_{n-1}(y) g_{L}(y)-g_{H}(y)\right\} d y \\
& -\int_{x^{*}}^{\bar{x}} \frac{\mu_{H} \mu_{L}}{\left[\mu_{H} \rho_{1}(y) \rho_{n-1}(y)+\gamma \mu_{L}\right]}\left\{\rho_{1}(y) \rho_{n-1}(y) g_{L}(y)-g_{H}(y)\right\} \Gamma\left(x^{*}\right) d y \\
& =\int_{x^{*}}^{\bar{x}} \frac{\mu_{L} \mu_{H}\left\{\mu_{H} v_{H} \rho_{1}(x) \rho_{n-1}(x)-\mu_{L}\left[(1-\gamma) \Gamma\left(x^{*}\right)-v_{L}\right]-\left[\mu_{H} \rho_{1}(y) \rho_{n-1}(y)+\gamma \mu_{L}\right] \Gamma\left(x^{*}\right)\right\}}{\left[\mu_{H} \rho_{1}(y) \rho_{n-1}(y)+\gamma \mu_{L}\right]^{2}} \\
& \left\{\rho_{1}(y) \rho_{n-1}(y) g_{L}(y)-g_{H}(y)\right\} d y \\
& =\int_{x^{*}}^{\bar{x}} \frac{\mu_{L} \mu_{H}}{\left[\mu_{H} \rho_{1}(y) \rho_{n-1}(y)+\gamma \mu_{L}\right]^{2}}\left\{\mu_{H} \rho_{1}(x) \rho_{n-1}(x)\left[v_{H}-\Gamma\left(x^{*}\right)\right]-\mu_{L}\left[\Gamma\left(x^{*}\right)-v_{L}\right]\right\} \\
& \left\{\rho_{1}(y) \rho_{n-1}(y) g_{L}(y)-g_{H}(y)\right\} d y \\
& =\int_{x^{*}}^{\bar{x}} \frac{\mu_{L} \mu_{H}}{\left[\mu_{H} \rho_{1}(y) \rho_{n-1}(y)+\gamma \mu_{L}\right]^{2}} \frac{\left[\mu_{L} \mu_{H}\left(v_{H}-v_{L}\right)\right]\left[\rho_{1}(x) \rho_{n-1}(x)-\rho_{1}\left(x^{*}\right) \rho_{n-1}\left(x^{*}\right)\right]}{\mu_{H} \rho_{1}\left(x^{*}\right) \rho_{n-1}\left(x^{*}\right)+\mu_{L}}
\end{aligned}
$$


$<0$.

$$
\left\{\rho_{1}(y) \rho_{n-1}(y) g_{L}(y)-g_{H}(y)\right\} d y
$$

Therefore, the seller's revenue is strictly decreasing in $\gamma$ given $x^{*}$, and Proposition 5 is proven.

\section{Appendix B: Experimental Instructions (translated from}

\section{Chinese)}

This is an experiment in the economics of market decision making. The experiment consists of two parts. Now we are implementing Part I of the experiment. Part II of the experiment will be implemented 7 days from now (in the same time slot) at the same place. You are invited to participate in both Part I and Part II. Several research organizations have provided funds for conducting this research. The instructions are simple, and if you follow them carefully and make good decisions you may earn a CONSIDERABLE AMOUNT OF MONEY, which will be PAID TO YOU IN CASH. You will receive your payments for both Part I and Part II at the end of Part II of the experiment.

In this experiment we will create a market in which you will act as a bidder of a fictitious commodity. There will be 17 trading periods, with the first two being practice periods with no monetary payoffs. In each trading period you will be paired with another bidder. A single unit of the commodity will be auctioned with the two of you as bidders. Your pairings will vary over periods and will remain anonymous.

\section{Values}

All values in the experiment will be in terms of experimental dollars. In each period the value of the auctioned item could be either 0 or 100 experimental dollars, with equal probability. The value of the auctioned item in one period has no impact on the value in the next period.

\section{Signals}

In each period each bidder will receive a private signal of the value of the auctioned item. If the value is 0 , then an urn with the following 45 numbered chips will be presented to you: 
$1,1,1,1,1,1,1,1,1$

$2,2,2,2,2,2,2,2$

$3,3,3,3,3,3,3$

$4,4,4,4,4,4$

$5,5,5,5,5$

$6,6,6,6$

$7,7,7$

8,8

9

As shown, we have one 9, two 8 s, three $7 \mathrm{~s}$, four $6 \mathrm{~s}$, five $5 \mathrm{~s}$, six $4 \mathrm{~s}$, seven $3 \mathrm{~s}$, eight $2 \mathrm{~s}$, and nine 1s, 45 chips altogether. You draw one chip from the urn and the number on the chip is your signal. If the value of the auctioned item is 0 , then the chance of receiving a signal of " 1 " is $9 / 45$, the chance receiving a signal of " 2 " is $8 / 45, \ldots$, the chance of receiving a signal of " 9 " is $1 / 45$.

If the value is 100 , then an urn with the following 45 numbered chips will be presented to you:

1

2, 2

$3,3,3$

$4,4,4,4$

$5,5,5,5,5$

$6,6,6,6,6,6$

$7,7,7,7,7,7,7$

$8,8,8,8,8,8,8,8$

$9,9,9,9,9,9,9,9,9$

As shown, we have nine 9s, eight 8 s, seven $7 \mathrm{~s}$, six $6 \mathrm{~s}$, five $5 \mathrm{~s}$, four $4 \mathrm{~s}$, three $3 \mathrm{~s}$, two $2 \mathrm{~s}$, one 1 , again a total of 45 chips. You draw one chip from the urn and the number on the chip is your signal. If the value of the auctioned item is 100 , then the chance of receiving a signal of " 1 " is $1 / 45$, the chance receiving a signal of " 2 " is $2 / 45, \ldots$, the chance of receiving a signal of " 9 " is $9 / 45$.

NOTE: Your signal has no impact on the other bidder's signal, but both have been drawn from the same urn containing the same 45 numbered chips. Note that once one chip is drawn to be the signal for one bidder, it is placed back in the urn before 
another chip is drawn to be the signal for the second bidder. In the first practice period, real urns will be presented to you. Starting from the second practice period and throughout the rest of the experiment, the signals will be generated by computer in exactly the same manner as described above. You should think of the computer as randomly selecting one of the two urns with equal probability. Subsequently the computer will draw one virtual chip from the selected virtual urn and show the number on that chip to you. This is your signal.

\section{Auction Procedure}

At the beginning of each period, bidders will not be told the value of the auctioned item. Instead, each bidder receives a private signal as described above. Then both bidders submit bids for the item. The bidder with the higher bid purchases the item and pays the lower amount that was bid by the other bidder. This generates a profit of

Profit $=($ Value of the item $)-($ the lower bid that the other bidder submitted $)$.

The bidder with the lower bid earns zero profit. In case the two bidders submit the same bid, the computer will determine who will buy the item though a random process that gives each bidder an equal chance of being selected. In this case, the bidder selected to purchase the item pays an amount equal to the identical bids submitted by the two bidders.

After the purchase, both bidders observe the actual value of the item.

(Note: The following section in \{\} describes the return policy in the FR (or HF/LF) treatment, and is not shown in the NR treatment.)

\section{$\{$ Return Policy\}}

\{In each period, after finding out the value of the item, the bidder who purchased it has the opportunity to return it to the experimenter. If the bidder returns the item, then the bidder gets back the payment for the item and forfeits the value of the item. (in HF/LF treatments: The bidder also needs to pay a handling fee of $20 / 5$ experimental dollars.) This generates a profit of 0 (or $-20 /-5$ in HF/LF treatments) for the period.\}

\section{Total Payoffs}


You will be given a starting capital credit balance of 225 experimental dollars which includes your show-up fee. The conversion rate from experimental dollars is 1 Yuan $=15$ experimental dollars. Any profit earned by you in the experiment will be added to this sum, and any losses incurred will be subtracted from this sum. The net balance of these transactions will be calculated and paid to you in CASH at the end of the experiment. The starting capital credit balance, and whatever subsequent profits you earn, permit you to suffer losses in one auction to be recouped in part or in total in later auctions. However, should your net balance at any time during the experiment drop to zero (or less), you will no longer be permitted to participate. Instead you earn zero and you'll be free to leave the auction.

IMPORTANT, your signals are strictly private information and are not to be revealed to anyone else. You are not to reveal your bids or profits, nor speak with other participants while the experiment is in progress. This is important for the validity of the experiment and your cooperation is required for continuing participation in the experiment.

Do you have any questions about the instructions or procedures? If you have a question, please raise your hand and one of us will come to you. 


\section{For Online Publication}

\section{Online Appendix: Proof for Proposition 1}

\section{Proof for Proposition 1}

In general, there are three different cases for an equilibrium bidding function regarding the winning bidder's return decision: NR (Never Return), AR (Always Return), and PR (Partial Return):

Case NR: $a \geq(1-\gamma) B(\bar{x})-v_{L}-c_{B}$. In this case, the winning bidder keeps the object all the time. This is because even if s/he pays the highest price $B(\bar{x})$ and discovers that $V=v_{L}$, s/he still does not want to return the object for a refund.

Case AR: $a \leq(1-\gamma) B(\underline{x})-v_{L}-c_{B}$. In this case, the winning bidder returns the object whenever $V=v_{L}$. This is because if $\mathrm{s} /$ he returns the object when paying the lowest price $B(\underline{x})$, s/he would definitely return it when $\mathrm{s} /$ he pays a higher price.

Case PR: $(1-\gamma) B(\underline{x})-v_{L}-c_{B}<a<(1-\gamma) B(\bar{x})-v_{L}-c_{B}$. In this case, the winning bidder's return decision when $V=v_{L}$ depends on the price s/he pays in the auction. If $(1-\gamma) B(z)-v_{L}-c_{B}>a, \mathrm{~s} /$ he would return the object; if $(1-\gamma) B(z)-$ $v_{L}-c_{B}<a, \mathrm{~s} /$ he would not return the object.

Below, we analyze the three cases in turn.

\section{Case NR: Never return}

We first characterize the symmetric equilibrium bidding function in the case where the winning bidder never returns the object after winning. Let $B^{1}(\cdot)$ denote the bidding function in this case. Consider buyer 1. Suppose that buyer 1's signal is $x$ and s/he pretends to have signal $\tilde{x}$ and bids $B^{1}(\tilde{x})$. Given that when the realization of the value is $v_{L}$, bidder 1 will keep the object if $\mathrm{s} /$ he wins, his/her expected surplus in the auction is given by:

$$
\begin{aligned}
& \Pi^{1}(x, \tilde{x}) \\
&= \operatorname{Pr}\left(V=v_{H} \mid x_{1}=x\right) E\left\{\left[V-B^{1}(z)\right] I\{z<\tilde{x}\} \mid x_{1}=x, V=v_{H}\right\} \\
&+\operatorname{Pr}\left(V=v_{L} \mid x_{1}=x\right) E\left\{\left[V-B^{1}(z)\right] I\{z<\tilde{x}\} \mid x_{1}=x, V=v_{L}\right\} \\
&= \mu_{H}(x) \int_{\underline{x}}^{\tilde{x}}\left[v_{H}-B^{1}(y)\right] d J_{H}(z)+\mu_{L}(x) \int_{\underline{x}}^{\tilde{x}}\left[v_{L}-B^{1}(y)\right] d J_{L}(z), \\
& \text { Online Appendix: page } 1
\end{aligned}
$$


where

$$
\begin{aligned}
& \mu_{H}(x) \equiv \operatorname{Pr}\left(V=v_{H} \mid x_{1}=x\right) \\
= & \frac{\operatorname{Pr}\left(x_{1}=x \mid V=v_{H}\right) \operatorname{Pr}\left(V=v_{H}\right)}{\operatorname{Pr}\left(x_{1}=x \mid V=v_{H}\right) \operatorname{Pr}\left(V=v_{H}\right)+\operatorname{Pr}\left(x_{1}=x \mid V=v_{L}\right) \operatorname{Pr}\left(V=v_{L}\right)} \\
= & \frac{f_{H}(x) \mu_{H}}{f_{H}(x) \mu_{H}+f_{L}(x) \mu_{L}},
\end{aligned}
$$

and where $\mu_{L}(x)=\operatorname{Pr}\left(V=v_{L} \mid x_{1}=x\right)=1-\mu_{H}(x)$. It is important to note that $\mu_{H}(x)$ is increasing in $x$ and $\mu_{L}(x)$ is decreasing in $x$. Therefore,

$$
\begin{aligned}
& \frac{\partial \Pi^{1}(x, \tilde{x})}{\partial \tilde{x}} \\
= & \mu_{H}(x)\left[v_{H}-B^{1}(\tilde{x})\right] j_{H}(\tilde{x})+\mu_{L}(x)\left[v_{L}-B^{1}(\tilde{x})\right] j_{L}(\tilde{x}) \\
= & {\left[\mu_{H}(x) j_{H}(\tilde{x})+\mu_{L}(x) j_{L}(\tilde{x})\right]\left[\frac{\mu_{H}(x) v_{H} j_{H}(\tilde{x})+\mu_{L}(x) v_{L} j_{L}(\tilde{x})}{\mu_{H}(x) j_{H}(\tilde{x})+\mu_{L}(x) j_{L}(\tilde{x})}-B^{1}(\tilde{x})\right] } \\
= & {\left[\mu_{H}(x) j_{H}(\tilde{x})+\mu_{L}(x) j_{L}(\tilde{x})\right]\left[\frac{\mu_{H} j_{H}(x) v_{H} j_{H}(\tilde{x})+\mu_{L} j_{(}(x) v_{L} j_{L}(\tilde{x})}{\mu_{H} j_{H}(x) j_{H}(\tilde{x})+\mu_{L} j_{L}(x) j_{L}(\tilde{x})}-B^{1}(\tilde{x})\right] } \\
= & {\left[\mu_{H}(x) j_{H}(\tilde{x})+\mu_{L}(x) j_{L}(\tilde{x})\right]\left[\frac{\mu_{H} v_{H} \rho_{1}(x) \rho_{n-1}(\tilde{x})+\mu_{L} v_{L}}{\mu_{H} \rho_{1}(x) \rho_{n-1}(\tilde{x})+\mu_{L}}-B^{1}(\tilde{x})\right] . }
\end{aligned}
$$

The first order condition (FOC) for this bidder's surplus maximization problem gives:

$$
\left.\frac{\partial \Pi^{1}(x, \tilde{x})}{\partial \tilde{x}}\right|_{\tilde{x}=x}=0 .
$$

Solving for $B^{1}(x)$, we have

$$
B^{1}(x)=\frac{\mu_{H} v_{H} \rho_{1}(x) \rho_{n-1}(x)+\mu_{L} v_{L}}{\mu_{H} \rho_{1}(x) \rho_{n-1}(x)+\mu_{L}} .
$$

The FOC is usually only a necessary condition. We shall show below that the FOC is also a sufficient condition for the above maximization problem. It is easy to check that $\frac{\mu_{H} v_{H} \rho_{1}(x) \rho_{n-1}(\tilde{x})+\mu_{L} v_{L}}{\mu_{H} \rho_{1}(x) \rho_{n-1}(\tilde{x})+\mu_{L}}$ is increasing in $x$. Therefore, given the bidding function defined in equation (10), the surplus function $\Pi^{1}(x, \tilde{x})$ is a unimodal function with the maximum at $\tilde{x}=x$; i.e., increasing for $\tilde{x} \leq x$ and decreasing for $\tilde{x} \geq x$. To see this, for $\tilde{x} \leq x$

$$
\begin{aligned}
& \frac{\partial \Pi^{1}(x, \tilde{x})}{\partial \tilde{x}} \\
= & {\left[\mu_{H}(x) f_{H}(\tilde{x})+\mu_{L}(x) f_{L}(\tilde{x})\right]\left[\frac{\mu_{H} v_{H} \rho_{1}(x) \rho_{n-1}(\tilde{x})+\mu_{L} v_{L}}{\mu_{H} \rho_{1}(x) \rho_{n-1}(\tilde{x})+\mu_{L}}-\frac{\mu_{H} v_{H} \rho_{1}(\tilde{x}) \rho_{n-1}(\tilde{x})+\mu_{L} v_{L}}{\mu_{H} \rho_{1}(\tilde{x}) \rho_{n-1}(\tilde{x})+\mu_{L}}\right] } \\
\geq & {\left[\mu_{H}(x) f_{H}(\tilde{x})+\mu_{L}(x) f_{L}(\tilde{x})\right]\left[\frac{\mu_{H} v_{H} \rho_{1}(\tilde{x}) \rho_{n-1}(\tilde{x})+\mu_{L} v_{L}}{\mu_{H} \rho_{1}(\tilde{x}) \rho_{n-1}(\tilde{x})+\mu_{L}}-\frac{\mu_{H} v_{H} \rho_{1}(\tilde{x}) \rho_{n-1}(\tilde{x})+\mu_{L} v_{L}}{\mu_{H} \rho_{1}(\tilde{x}) \rho_{n-1}(\tilde{x})+\mu_{L}}\right] } \\
= & 0,
\end{aligned}
$$


and for $\tilde{x} \geq x$,

$$
\begin{aligned}
& \frac{\partial \Pi^{1}(x, \tilde{x})}{\partial \tilde{x}} \\
= & {\left[\mu_{H}(x) f_{H}(\tilde{x})+\mu_{L}(x) f_{L}(\tilde{x})\right]\left[\frac{\mu_{H} v_{H} \rho_{1}(x) \rho_{n-1}(\tilde{x})+\mu_{L} v_{L}}{\mu_{H} \rho_{1}(x) \rho_{n-1}(\tilde{x})+\mu_{L}}-\frac{\mu_{H} v_{H} \rho_{1}(\tilde{x}) \rho_{n-1}(\tilde{x})+\mu_{L} v_{L}}{\mu_{H} \rho_{1}(\tilde{x}) \rho_{n-1}(\tilde{x})+\mu_{L}}\right] } \\
\leq & {\left[\mu_{H}(x) f_{H}(\tilde{x})+\mu_{L}(x) f_{L}(\tilde{x})\right]\left[\frac{\mu_{H} v_{H} \rho_{1}(\tilde{x}) \rho_{n-1}(\tilde{x})+\mu_{L} v_{L}}{\mu_{H} \rho(\tilde{x}) \rho(\tilde{x})+\mu_{L}}-\frac{\mu_{H} v_{H} \rho_{1}(\tilde{x}) \rho_{n-1}(\tilde{x})+\mu_{L} v_{L}}{\mu_{H} \rho_{1}(\tilde{x}) \rho_{n-1}(\tilde{x})+\mu_{L}}\right] } \\
= & 0 .
\end{aligned}
$$

Therefore, $\tilde{x}=x$ is indeed optimal and the sufficiency of the FOC for the maximization is confirmed. Of course, for the above bidding function to be an equilibrium, we need to guarantee that the winner never wants to return the object. Note that the bidding function is increasing. The condition of no return is equivalent to

$$
\frac{v_{L}+a+c_{B}}{1-\gamma} \geq B^{1}(\bar{x}) \Leftrightarrow \frac{v_{L}+a+c_{B}}{1-\gamma} \geq \Gamma(\bar{x}) .
$$

Case AR: Always return when $V=v_{L}$

In this case, the winning bidder always returns the object when $V=v_{L}$. Given this, buyer 1's surplus when s/he pretends to have signal $\tilde{x}$ is given by

$$
\begin{aligned}
& \Pi^{2}(x, \tilde{x}) \\
= & \operatorname{Pr}\left(V=v_{H} \mid x_{1}=x\right) E\left\{\left[V-B^{2}(z)\right] I\{y<\tilde{x}\} \mid x_{1}=x, V=v_{H}\right\} \\
& +\operatorname{Pr}\left(V=v_{L} \mid x_{1}=x\right)\left[-E\left\{\gamma B^{2}(z) I\{y<\tilde{x}\} \mid x_{1}=x, V=v_{L}\right\}-a-c_{B}\right] \\
= & \mu_{H}(x) \int_{\underline{x}}^{\tilde{x}}\left[v_{H}-B^{2}(z)\right] d J_{H}(z)-\mu_{L}(x) \int_{\underline{x}}^{\tilde{x}}\left[a+\gamma B^{2}(z)+c_{B}\right] d J_{L}(z) .
\end{aligned}
$$

Taking the derivative with respect to $\tilde{x}$, we have

$$
\begin{aligned}
& \frac{\partial \Pi^{2}(x, \tilde{x})}{\partial \tilde{x}} \\
= & \mu_{H}(x)\left[v_{H}-B^{2}(\tilde{x})\right] j_{H}(\tilde{x})-\mu_{L}(x)\left[a+c_{B}+\gamma B^{2}(\tilde{x})\right] j_{L}(\tilde{x}) \\
= & {\left[\mu_{H}(x) j_{H}(\tilde{x})+\gamma \mu_{L}(x) j_{L}(\tilde{x})\right]\left[\frac{\mu_{H}(x) v_{H} j_{H}(\tilde{x})-\mu_{L}(x)\left(a+c_{B}\right) j_{L}(\tilde{x})}{\mu_{H}(x) j_{H}(\tilde{x})+\gamma \mu_{L}(x) j_{L}(\tilde{x})}-B^{2}(\tilde{x})\right] } \\
= & {\left[\mu_{H}(x) j_{H}(\tilde{x})+\gamma \mu_{L}(x) j_{L}(\tilde{x})\right]\left[\frac{\mu_{H} j_{H}(x) v_{H} j_{H}(\tilde{x})-\mu_{L} j_{L}(x)\left(a+c_{B}\right) j_{L}(\tilde{x})}{\mu_{H} j_{H}(x) j_{H}(\tilde{x})+\gamma \mu_{L} j_{L}(x) j_{L}(\tilde{x})}-B^{2}(\tilde{x})\right] } \\
= & {\left[\mu_{H}(x) j_{H}(\tilde{x})+\gamma \mu_{L}(x) j_{L}(\tilde{x})\right]\left[\frac{\mu_{H} v_{H} \rho_{1}(x) \rho_{n-1}(\tilde{x})-\mu_{L}\left(a+c_{B}\right)}{\mu_{H} \rho_{1}(x) \rho_{n-1}(\tilde{x})+\gamma \mu_{L}}-B^{2}(\tilde{x})\right] . }
\end{aligned}
$$

The first order condition for bidder 1's surplus maximization problem is

$$
\left.\frac{\partial \Pi^{2}(x, \tilde{x})}{\partial \tilde{x}}\right|_{\tilde{x}=x}=0
$$

Solving for $B^{2}(x)$, we have

$$
\begin{gathered}
B^{2}(x)=\frac{\mu_{H} v_{H} \rho_{1}(x) \rho_{n-1}(x)-\mu_{L}\left(a+c_{B}\right)}{\mu_{H} \rho_{1}(x) \rho_{n-1}(x)+\gamma \mu_{L}} . \\
\text { Online Appendix: page } 3
\end{gathered}
$$


The FOC is usually only a necessary condition. It is easy to check that $\frac{\mu_{H} v_{H} \rho_{1}(x) \rho_{n-1}(\tilde{x})-\mu_{L}\left(a+c_{B}\right)}{\mu_{H} \rho_{1}(x) \rho_{n-1}(\tilde{x})+\gamma \mu_{L}}$ is increasing in $x$. Similar to the argument in Case NR, the surplus function $\Pi^{2}(x, \tilde{x})$ is a unimodal function with maximum at $\tilde{x}=x$ when using the bidding function defined in equation (11). As a result, the sufficiency of the FOC for the maximization is confirmed.

Again, for this bidding function to be an equilibrium, the condition for "always returning" has to be satisfied. Given that the bidding function is increasing, this condition is equivalent to

$$
\begin{aligned}
& \frac{v_{L}+a+c_{B}}{1-\gamma} \leq B^{2}(\underline{x}) \\
\Leftrightarrow & \frac{v_{L}+a+c_{B}}{1-\gamma} \leq \frac{\mu_{H} v_{H} \rho_{1}(x) \rho_{n-1}(x)-\mu_{L}\left(a+c_{B}\right)}{\mu_{H} \rho_{1}(x) \rho_{n-1}(x)+\gamma \mu_{L}} \\
\Leftrightarrow & \frac{\mu_{L}\left(v_{L}+a+c_{B}\right)}{\mu_{L}(1-\gamma)} \leq \frac{\mu_{H} v_{H} \rho_{1}(x) \rho_{n-1}(x)-\mu_{L}\left(a+c_{B}\right)}{\mu_{H} \rho_{1}(x) \rho_{n-1}(x)+\gamma \mu_{L}} \\
\Leftrightarrow & \mu_{L}\left(v_{L}+a+c_{B}\right)\left[\mu_{H} \rho_{1}(x) \rho_{n-1}(x)+\gamma \mu_{L}\right] \\
& \quad \leq \mu_{L}(1-\gamma)\left[\mu_{H} v_{H} \rho_{1}(x) \rho_{n-1}(x)-\mu_{L}\left(a+c_{B}\right)\right] \\
\Leftrightarrow & \mu_{L}\left(v_{L}+a+c_{B}\right)\left[\mu_{H} \rho_{1}(x) \rho_{n-1}(x)+\gamma \mu_{L}\right]+\mu_{L}\left(v_{L}+a+c_{B}\right)\left[\mu_{L}(1-\gamma)\right] \\
& \leq \mu_{L}(1-\gamma)\left[\mu_{H} v_{H} \rho_{1}(x) \rho_{n-1}(x)-\mu_{L}\left(a+c_{B}\right)\right]+\mu_{L}\left(v_{L}+a+c_{B}\right)\left[\mu_{L}(1-\gamma)\right] \\
\Leftrightarrow & \mu_{L}\left(v_{L}+a+c_{B}\right)\left[\mu_{H} \rho_{1}(x) \rho_{n-1}(x)+\mu_{L}\right] \\
& \quad \leq \mu_{L}(1-\gamma)\left[\mu_{H} v_{H} \rho_{1}(x) \rho_{n-1}(x)+\mu_{L} V_{L}\right] \\
\Leftrightarrow & \frac{v_{L}+a+c_{B}}{1-\gamma} \leq \Gamma(\underline{x})
\end{aligned}
$$

\section{Case PR: Cutoff rule when $V=v_{L}$}

In this case, there is an endogenously determined cutoff in the winning bidder's return decision. We denote this cutoff as $x^{*}$. Buyer 1's surplus by pretending to have signal $\tilde{x}$ is given by

$$
\begin{aligned}
& \Pi(x, \tilde{x}) \\
& = \begin{cases}\Pi^{1}(x, \tilde{x}), & \text { if } \quad \tilde{x} \leq x^{*} ; \\
\mu_{H}(x)\left\{\int_{\underline{x}}^{\tilde{x}}\left[v_{H}-B(z)\right] d j_{H}(z)\right\} & \\
+\mu_{L}(x)\left\{\int_{\underline{x}}^{x^{*}}\left[v_{L}-B(z)\right] d j_{L}(z)-\int_{x^{*}}^{\tilde{x}}\left[a+\gamma B(y)+c_{B}\right] d j_{L}(z)\right\}, & \text { if } \quad \tilde{x} \geq x^{*} .\end{cases}
\end{aligned}
$$

Note that the above function is continuous. Taking the derivative of the above with respect to $\tilde{x}$, we have 


$$
\frac{\partial \Pi(x, \tilde{x})}{\partial \tilde{x}}= \begin{cases}\frac{\partial \Pi^{1}(x, \tilde{x})}{\partial \tilde{x}}, & \text { if } \tilde{x} \leq x^{*} \\ \frac{\partial \Pi^{2}(x, \tilde{x})}{\partial \tilde{x}}, & \text { if } \tilde{x} \geq x^{*}\end{cases}
$$

Although $\Pi(x, \tilde{x}) \neq \Pi^{2}(x, \tilde{x})$ when $\tilde{x} \geq x^{*}$, we have $\frac{\partial \Pi(x, \tilde{x})}{\partial \tilde{x}}=\frac{\partial \Pi^{2}(x, \tilde{x})}{\partial \tilde{x}}$. From the first order condition, we can derive the bidding function as follows:

$$
B(x)= \begin{cases}B^{1}(x)=\frac{\mu_{H} v_{H} \rho_{1}(x) \rho_{n-1}(x)+\mu_{L} v_{L}}{\mu_{H} \rho_{1}(x) \rho_{n-1}(x)+\mu_{L}}, & \text { if } x \leq x^{*} ; \\ B^{2}(x)=\frac{\mu_{H} v_{H} \rho_{1}(x) \rho_{n-1}(x)-\mu_{L}\left(a+c_{B}\right)}{\mu_{H} \rho_{1}(x) \rho_{n-1}(x)+\gamma \mu_{L}}, & \text { if } x \geq x^{*} .\end{cases}
$$

Note that $x^{*}$ is determined by

$$
B^{1}\left(x^{*}\right)=\frac{v_{L}+a+c_{B}}{1-\gamma},
$$

i.e.,

$$
\Gamma\left(x^{*}\right)=\frac{v_{L}+a+c_{B}}{1-\gamma} .
$$

Note that functions $B^{2}(x)$ and $B^{1}(x)$ cross each other at $x^{*}$. Now consider the sufficient condition. Given the bidding function (13), from the proof in Cases 1 and 2, we know that $\Pi^{1}(x, \tilde{x})$ is a unimodal function with the maximum at $\tilde{x}=x$ when $\tilde{x} \leq x^{*}$; and $\Pi^{2}(x, \tilde{x})$ is a unimodal function with the maximum at $\tilde{x}=x$ when $\tilde{x} \geq x^{*}$. We shall show that $\Pi(x, \tilde{x})$ is also a unimodal function with maximum at $\tilde{x}=x$. Consider $x \leq x^{*}$, for example. For $\underline{x} \leq \tilde{x} \leq x$, the payoff is increasing in $\tilde{x}$ from the first formula of (12). For $x \leq \tilde{x} \leq x^{*}$, the payoff is decreasing in $\tilde{x}$ from the first formula of (12). For $x^{*} \leq \tilde{x} \leq \bar{x}$, the payoff is decreasing in $\tilde{x}$ from the second formula of (12). Therefore, $\Pi(x, \tilde{x})$ achieves its maximal value at $\tilde{x}=x$. Similar arguments can be applied to the case of $x \geq x^{*}$. Thus, the sufficient condition for the maximization is satisfied.

In this equilibrium, when $V=v_{L}$, the winning bidder returns the object if $\mathrm{s} / \mathrm{he}$ pays too much, and keeps the object otherwise. For this to happen, $\gamma$ has to satisfy the following condition:

$$
B^{1}(\bar{x})>\frac{v_{L}+a+c_{B}}{1-\gamma}>B^{1}(\underline{x}) \text {, or, } \Gamma(\bar{x})>\frac{v_{L}+a+c_{B}}{1-\gamma}>\Gamma(\underline{x}) .
$$

Furthermore, the intervals for the above three cases do not overlap with each other and they cover the entire range of $a$ and $\gamma$. Thus we can conclude that a unique symmetric perfect Bayesian Nash equilibrium exists for any value of $a$ and $\gamma$.

Proposition 1 simply summarizes all the situations. 